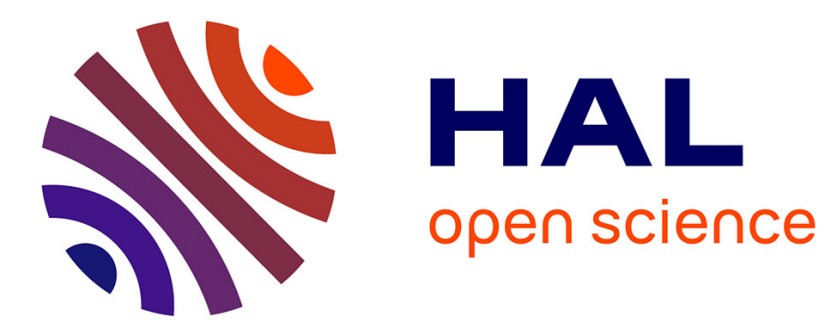

\title{
Scale-Dependent and Example-Based Stippling
}

Domingo Martín, Germán Arroyo, M. Victoria Luzón, Tobias Isenberg

\section{To cite this version:}

Domingo Martín, Germán Arroyo, M. Victoria Luzón, Tobias Isenberg. Scale-Dependent and Example-Based Stippling. Computers and Graphics, 2011, 35 (1), pp.160-174. 10.1016/j.cag.2010.11.006 . hal-00781505

\section{HAL Id: hal-00781505 \\ https://hal.inria.fr/hal-00781505}

Submitted on 27 Jan 2013

HAL is a multi-disciplinary open access archive for the deposit and dissemination of scientific research documents, whether they are published or not. The documents may come from teaching and research institutions in France or abroad, or from public or private research centers.
L'archive ouverte pluridisciplinaire $\mathbf{H A L}$, est destinée au dépôt et à la diffusion de documents scientifiques de niveau recherche, publiés ou non, émanant des établissements d'enseignement et de recherche français ou étrangers, des laboratoires publics ou privés. 


\title{
Scale-Dependent and Example-Based Grayscale Stippling
}

\author{
Domingo Martín ${ }^{1}$ \\ Germán Arroyo $^{1}$ \\ M. Victoria Luzón ${ }^{1}$ \\ Tobias Isenberg 2,3 \\ ${ }^{1}$ University of Granada, Spain \\ ${ }^{2}$ University of Groningen, The Netherlands \\ ${ }^{3}$ DIGITEO \& CNRS/INRIA, France
}

\begin{abstract}
We present an example-based approach to synthesizing stipple illustrations for static 2D images that produces scaledependent results appropriate for an intended spatial output size and resolution. We show how treating stippling as a grayscale process allows us to both produce on-screen output and to achieve stipple merging at medium tonal ranges. At the same time we can also produce images with high spatial and low color resolution for print reproduction. In addition, we discuss how to incorporate high-level illustration considerations into the stippling process based on discussions with and observations of a stipple artist. Also, certain features such as edges can be extracted and used to control the placement of dots to improve the result. The implementation of the technique is based on a fast method for distributing dots using halftoning and can be used to create stipple images interactively. We describe both a GPU implementation of the basic algorithm that creates stipple images in real-time for large images and an extended CPU method that allows a finer control of the output at interactive rates.
\end{abstract}

Keywords: Stippling, high-quality rendering, scale-dependent NPR, example-based techniques, illustrative visualization

\section{Introduction}

Stippling is a traditional pen-and-ink technique that is popular for creating illustrations in many domains. It relies on placing stipples (small dots that are created with a pen) on a medium such as paper such that the dots represent shading, material, and structure of the depicted objects. Stippling is frequently used by professional illustrators for creating illustrations, for example, in archeology (e.g., see Figure 2), entomology, ornithology, and botany.

One of the essential advantages of stippling that it shares with other pen-and-ink techniques is that it can be used in the common bilevel printing process. By treating the stipple dots as completely black marks on a white background they can easily be reproduced without losing spatial precision due to halftoning artifacts. This property and the simplicity of the dot as the main rendering element has lead to numerous approaches to synthesize stippling within Non-Photorealistic Rendering (NPR), describing techniques for distributing stipple dots such that they represent a given tone. Unfortunately, computergenerated stippling sometimes creates distributions with artifacts such as unwanted lines, needs a lot of computation power due to the involved computational complexity of the approaches, cannot re-create the merging of stipple dots in middle tonal ranges that characterizes many handdrawn examples, or produces output with dense stipple points unlike those of hand-drawn illustrations.

To address these issues, our goal is to realize a stippling process for 2D images (see example result in Figure 1) that is easy to implement, that considers the whole process of hand-made stippling, and that takes scale and output

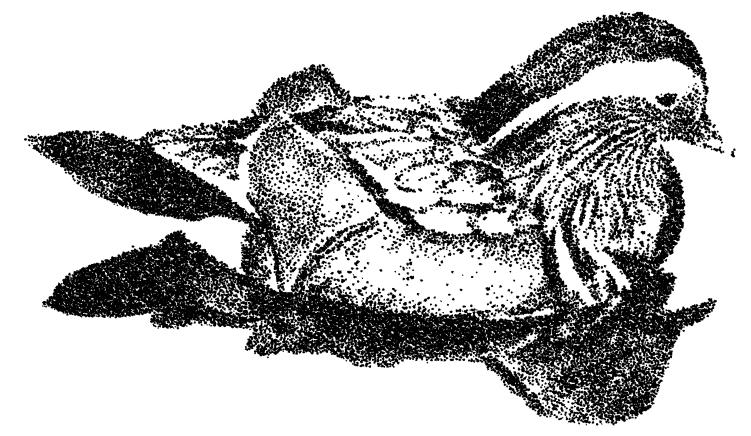

Figure 1: An example stipple image created with our technique.

devices into account. For this purpose we do not consider stippling to always be a black-and-white technique, in contrast to previous NPR approaches (e.g., [1]). In fact, we use the grayscale properties of hand-made stipple illustrations to inform the design of a grayscale stipple process. This different approach lets us solve not only the stipple merging problem but also lets us create output adapted to the intended output device. To summarize, our paper makes the following contributions:

- an analysis of high-level processes involved in stippling and a discussion on how to support these using image-processing,

- a method for example-based stippling in which the stipple dot placement is based on halftoning,

- the scale-dependent treatment of scanned stipple dot examples, desired output, and stipple placement, 
- a grayscale stippling process that can faithfully reproduce the merging of stipples at middle tonal ranges,

- the enabling of both print output in black-and-white and on-screen display in gray or tonal scales at the appropriate spatial and color resolutions,

- an optional special treatment of image edges that improves the control of the placement of stipples,

- a technique that is easy to implement and permits the interactive creation of stipple images,

- a description of a GPU implementation of the basic technique, and

- a comparative analysis of the stipple dot placement statistics for both the hand-drawn example and generated synthetic stipple images.

This article is an extended version of a paper [2] published at NPAR 2010. The remainder is structured as follows. First we review related work in the context of computer-generated stippling in Section 2. Next, we analyze hand-made stippling in Section 3, both with respect to high-level processes performed by the illustrator and low-level properties of the stipple dots. Based on this analysis we describe our scale-dependent grayscale stippling process in Section 4. We analyze the performance and describe a GPU implementation in Section 5, before discussing the results in Section 6 . We conclude the paper and suggest some ideas for future work in Section 7.

\section{Related Work}

Pen-and-ink rendering and, specifically, computer-generated stippling are well-examined areas within NPR. Many approaches exist to both replicating the appearance of hand-drawn stipple illustrations and using stippling within new contexts such as animation. In the following discussion we distinguish between stipple rendering based on 3D models such as boundary representations or volumetric data on the one side and stippling that uses 2D pixel images as input on the other.

The variety of types of $3 \mathrm{D}$ models used in computer graphics is also reflected in the diversity of stipple rendering approaches designed for them. There exist techniques for stippling of volume data [3, 4] typically aimed at visualization applications, stipple methods for implicit surfaces $[5,6]$, point-sampled surfaces [7, 8], and stippling of polygonal surfaces $[9,10]$. The placement of stipple dots in 3D space creates a unique challenge for these techniques because the viewer ultimately perceives the point distribution on the 2D plane. Related to this issue, animation of stippled surfaces $[11,12]$ presents an additional challenge as the stipples have to properly move with the changing object surface to avoid the shower-door effect. A special case of stippling of 3D models is the computation in a geometry-image domain [13] where the stippling is computed on a 2D geometry image onto which the 3D surface is mapped.

While Yuan et al. [13] map the computed stipples back onto the 3D surface, many approaches compute stippling only on 2D pixel images. The challenge here is to achieve an evenly spaced distribution that also reflects the gray value of the image to be represented, an optimization problem within stroke-based rendering [14]. One way to achieve a desired distribution is Lloyd's method [15, 16] that is based on iteratively computing the centroidal Voronoi diagram (CVD) of a point distribution. Deussen et al. [17] apply this technique to locally adjust the point spacing through interactive brushes, starting from initial point distribution-generated, e. g., by random sampling or halftoning-in which the point density reflects the intended gray values. The interactive but local application addresses a number of problems: the computational complexity of the technique as well as the issue that automatically processing the entire image would simply lead to a completely evenly distributed set of points. Thus, to allow automatic processing while maintaining the desired density, Secord [18] uses weighted Voronoi diagrams to reflect the intended local point density. A related way of achieving stipple placement was explored by Schlechtweg et al. [19] using a multi-agent system whose RenderBots evaluate their local neighborhood and try to move such that they optimize spacing to nearby agents with respect to the desired point density.

Besides evenly spaced distributions it is sometimes desirable to achieve different dot patterns. For instance, Mould [20] employs a distance-based path search in a weighted regular graph that ensures that stipple chains are placed along meaningful edges in the image. In another example, Kim et al. [21] use a constrained version of Lloyd's method to the arrange stipples along offset lines to illustrate images of faces. However, in most cases the distribution should not contain patterns such as chains of stipple points-professional illustrators specifically aim to avoid these artifacts. Thus, Kopf et al. [22] use Wangtiling to arrange stipple tiles in large, non-repetitive ways and show how to provide a continuous level of detail while maintaining blue noise properties. This means that one can zoom into a stipple image with new points continuously being added to maintain the desired point density.

In addition to stipple placement, another issue that has previously been addressed is the shape of stipple points. While most early methods use circles or rounded shapes to represent stipples $[17,18]$, several techniques have since been developed for other shapes, adapting Lloyd's method accordingly [23], using a probability density function [24], or employing spectral packing [25].

It is also interesting to examine the differences between computer-generated stipple images and hand-drawn examples. For example, Isenberg et al. [26] used an ethnographic pile-sorting approach to learn what people thought about both and what differences they perceive. They found that both the perfectly round shapes of stipple 


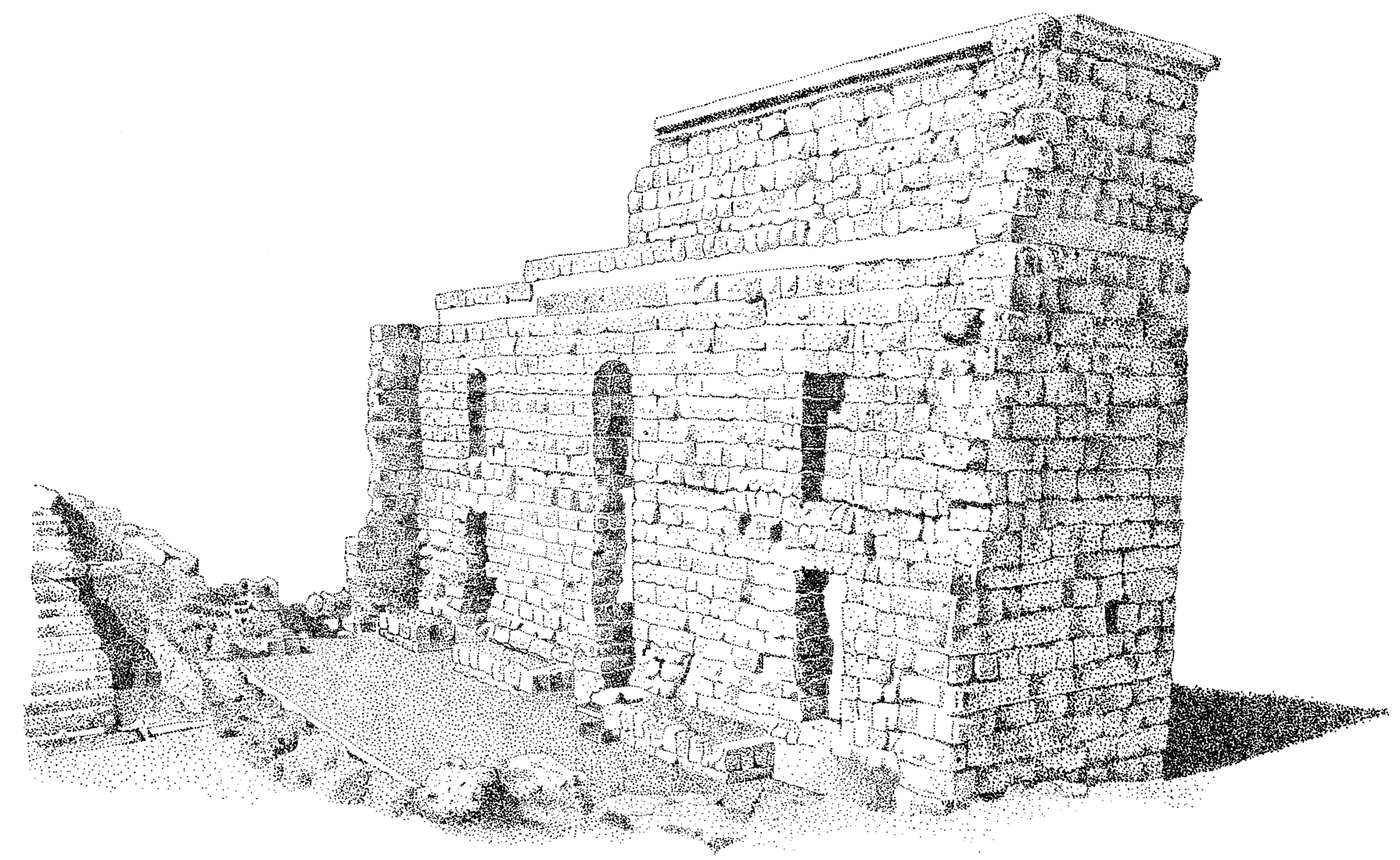

Figure 2: Hand-drawn stipple image by illustrator Elena Piñar of the Roman theater of Acinipo in Ronda, Málaga (originally on A4 paper). This image is (C) 2009 Elena Piñar, used with permission.

dots and the artifacts in placing them can give computergenerated images away as such, but also that people still valued them due to their precision and detail. Looking specifically at the statistics of stipple distributions, Maciejewski et al. [27] quantified these differences with statistical texture measures and found that, for example, computer-generated stippling exhibits an undesired spatial correlation away from the stipple points and a lack of correlation close to them. This lead to the exploration of example-based stippling, for instance, by Kim et al. [1]. They employ the same statistical evaluation as Maciejewski et al. [27] and use it to generate new stipple distributions that have the same statistical properties as hand-drawn examples. By then placing scanned stipple dots onto the synthesized positions Kim et al. [1] are able to generate convincing stipple illustrations. However, because the technique relies on being able to identify the centers of stipple points in the hand-drawn examples it has problems with middle tonal ranges because there stipple dots merge into larger conglomerates.

This issue of stipple merging in the middle tonal ranges is one problem that remains to be solved. In addition, while computer-generated stippling thus far has addressed stipple dot placement, stipple dot shapes, and animation, other aspects such as how to change an input image to create more powerful results (i. e., how to interpret the input image) have not yet been addressed.

\section{Analysis of Hand-Drawn Stippling}

To inform our technical approach for generating highquality computer-generated stipple illustrations, we start by analyzing the process professional stipple illustrators perform when creating a drawing. For this purpose we involved a professional stipple artist and asked her to explain her approach/process using the example illustration shown in Figure 2. From this analysis we extract a number of specific high-level processes that are often employed by professional stipple artists that go beyond simple dot placement and use of specific dot shapes. We discuss these in Section 3.1 before analyzing the low-level properties of stipple dots in Section 3.2 that guide our synthesis process.

\subsection{High-Level Processes}

The manual stipple process has previously been analyzed to inform computer-generated stippling. As part of this analysis and guided by literature on scientific illustration (e. g., [28]), researchers identified an even distribution of stipple points as one of the major goals (e.g., $[17,18]$ ) as well as the removal of artifacts (e.g., [22, 27]). Also, Kim et al. [1] noted the use of tone maps by illustrators to guide the correct reproduction of tone. While these aspects of stippling concentrate on rather low-level properties, there are also higher-level processes that stipple artists often employ in their work. Artists apply prior 


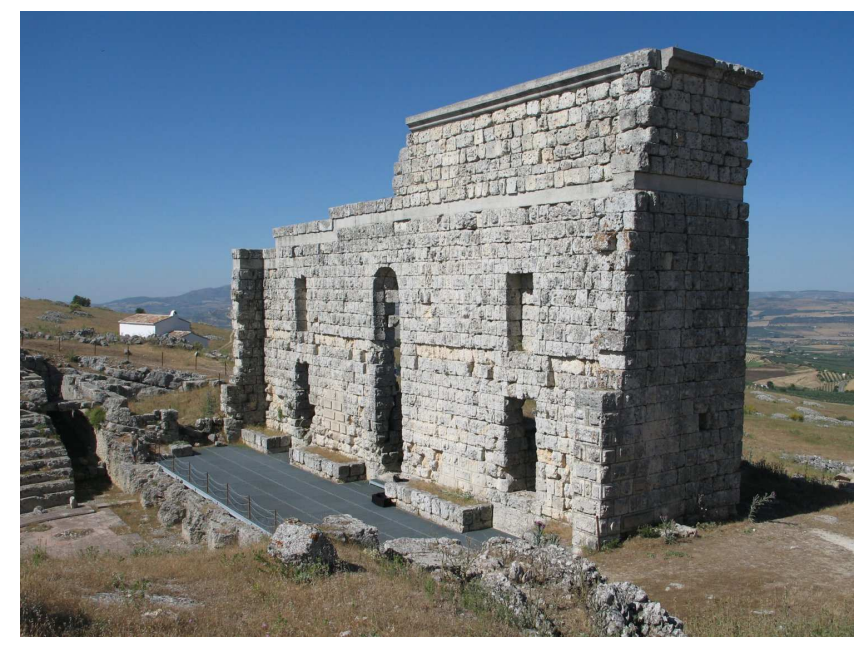

(a) Original photograph, Roman theater of Acinipo in Ronda, Málaga.

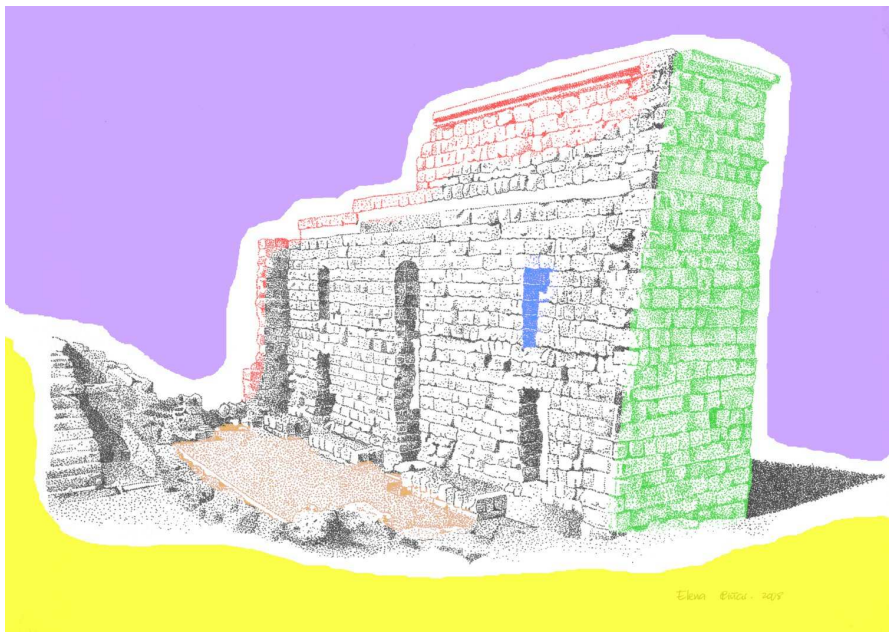

(b) Interpreted regions.

Figure 3: Original photograph and interpreted regions for Figure 2.

knowledge about good practices, knowledge about shapes and properties of the depicted objects, knowledge about the interaction of light with surfaces, and knowledge about the goal of the illustration. This leads to an interpretation of the original image or scene, meaning that stippling goes beyond an automatic and algorithmic tonal reproduction.

To explore these processes further we asked Elena Piñar, a professional illustrator, to create a stipple illustration (Figure 2) from a digital photo (Figure 3(a)). We observed and video-recorded her work on this illustration and also met with her afterwards to discuss her work and process. In this interview we asked her to explain the approach she took and the techniques she employed. From this conversation with her we could identify the following higher-level processes (see Figure 3 for a visual explanation with respect to the hand-made illustration in Figure 2 and photograph in Figure 3(a)). While this list is not comprehensive, according to Elena Piñar it comprises the most commonly used and most important techniques (some of these are mentioned, e. g., by Guptill [29]). Also, each artist has his or her own set of techniques as part of their own personal style.

Abstraction: One of the most commonly used techniques is removing certain parts or details in the image to focus the observer's attention on more important areas. In our example, the sky and some parts of the landscape have been fully removed (shown in violet in Figure 3(b)). In addition, removing areas contributes to a better image contrast.

Increase in contrast: Some parts of the original color image exhibit a low level of contrast, reducing their readability when stippled. To avoid this problem illustrators increase the contrast in such regions (green area) through global and local evaluation of lightness, enhancing the detail where necessary.
Irregular but smoothly shaped outlines: If objects in an image are depicted with a regular or rectilinear shape they are often perceived as being man-made. To avoid this impression for natural shapes, stipple artists eliminate parts of these objects to produce an irregular form and add a tonal gradient (yellow).

Reduction of complexity: It is not always possible to remove all unimportant areas. In these cases the complexity or amount of detail is reduced. This effect is shown in orange in Figure 3(b): the artist has removed some small parts that do not contribute to the illustration's intended message.

Additional detail: As visible in the red areas in Figure 3(b), some parts that are not (or not clearly) visible in the original are still shown in the illustration. Here, the illustrator has enhanced details of the rocks based on her prior knowledge.

Inversion: Sometimes artists convert very dark zones or edges into very clear ones to improve the contrast. This technique is applied subjectively to specific parts of the drawing rather than to the image as a whole. In the hand-made stippled drawing the cracks between rocks are shown in white while they are black in the original photograph (blue in Figure 3(b)).

Despite the fact that these high-level processes are an integral part of hand-drawn stipple illustration, computergenerated stippling techniques have largely concentrated on dot placement and dot shapes. This is understandable as these low-level processes can be automated while the higher-level processes to a large degree rely on human intelligence and sense of aesthetics. To be able to incorporate higher-level interpretations of images, therefore, we manually apply global and local image processing operations to the input image (Figure 4). Instead of directly using a gray-level input image (Figure 4(a)) we first apply 


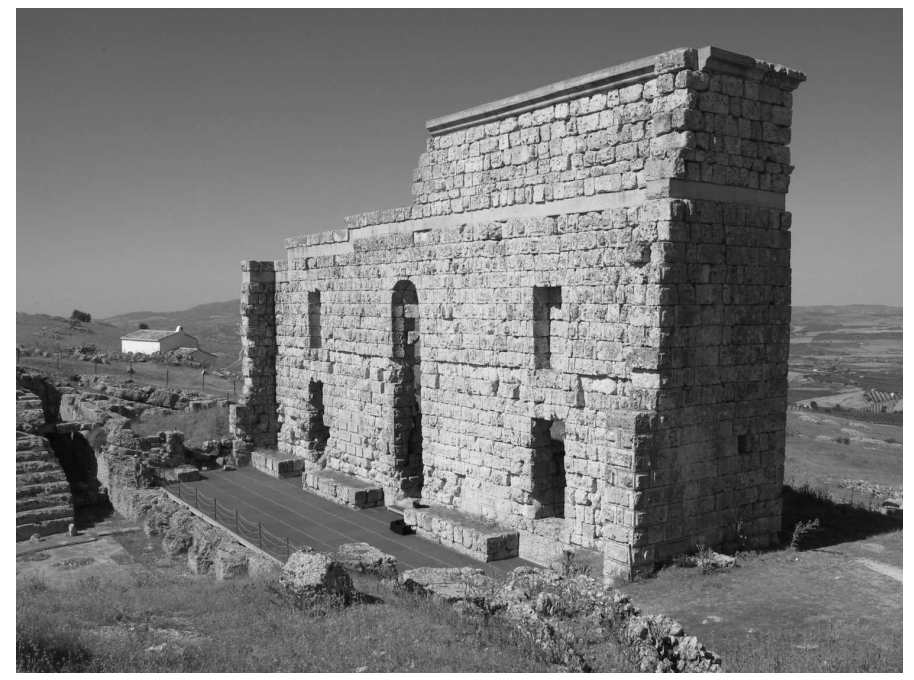

(a) Grayscale version of Figure 3(a).

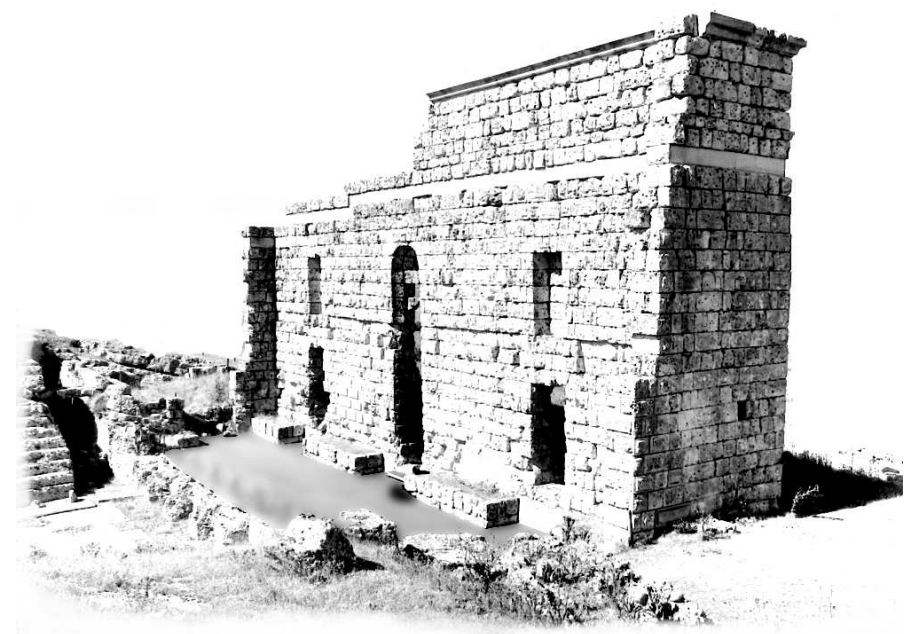

(b) Adjusting global contrast and local detail of (a).

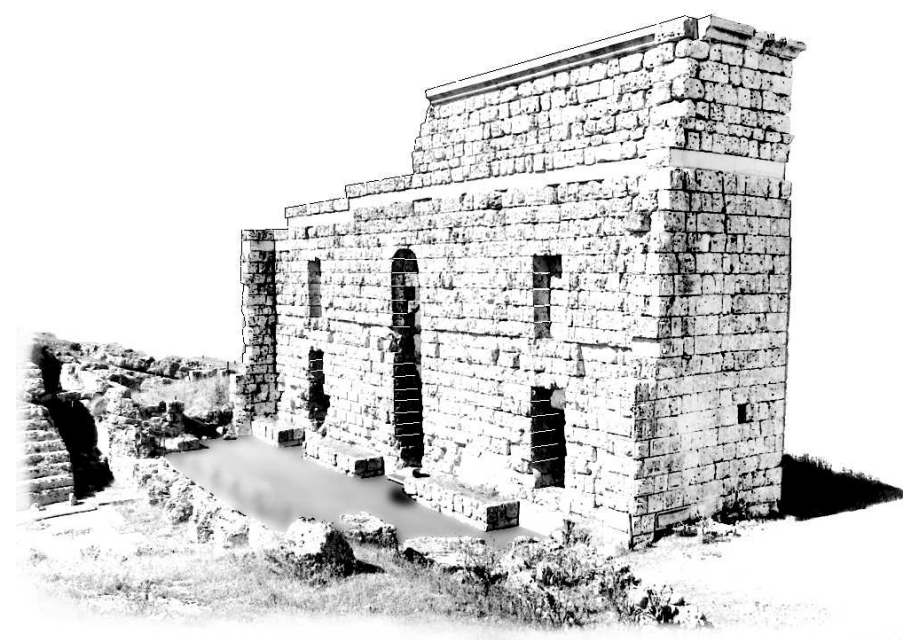

(c) Manually added edges, inversion, local contrast.

Figure 4: Deriving the helper image to capture the high-level stippling processes. pre-processing to accommodate the identified high-level processes. Following the list of processes given above (see Figure 4(b)), we remove non-relevant parts from the image such as the sky and parts of the background. Also, we increase the contrast globally but also increase the brightness of some regions locally. Next, we locally delete parts of natural objects and smooth the border of these regions. To reduce the complexity of certain parts such as the metal grid we select these regions and apply a large degree of blur. Adding elements based on previous knowledge typically requires the artist painting into the image. Some additional information, however, can be added with algorithmic support, in particular edges that border regions that are similar in brightness such as the top borders of the ruin. We support this by either extracting an edge image from the original color image, taking only regions into account that have not been deleted in Figure 4(b) and adding them to the helper image. Alternatively, artists can manually draw the necessary edges as shown in Figure 4(c). Finally, inversion can be achieved by also manually drawing the intended inverted edges as white lines into the touched-up grayscale image (see Figure 4(c)).

While this interactive pre-processing could be included into a comprehensive stipple illustration tool, we apply the manipulations using a regular image processing suite (e. g., Adobe Photoshop or GIMP). This allows us to make use of a great variety of image manipulation tools and effects to give us freedom to achieve the desired effects. The remainder of the process, on the other hand, is implemented in a dedicated tool. Before discussing our algorithm in detail, however, we first discuss some lowlevel aspects of hand-drawn stipple dots that are relevant for the approach.

\subsection{Low-Level Properties of Stipple Dots}

Stipple dots and their shapes have been analyzed and used in many previous computer stippling techniques, inspired by the traditional hand-drawn stippling. Hodges [28] notes that each dot should have a purpose and that dots should not become dashes. In computer-generated stippling, therefore, dots have typically been represented as circular or rounded shapes ${ }^{1}$ or pixels that are placed as black elements on a white ground. However, each use of a pen to place a dot creates a unique shape (e.g., Figure 5) which is partially responsible for the individual characteristics of a hand-made stipple illustration. Thus, recent computer-generated stippling employed scans of dots from hand-made illustrations to better capture the characteristics of hand-drawn stippling [1].

We follow a similar approach and collected a database of stipple points from a high-resolution scan of a handdrawn original illustration, a sample of which is shown in

\footnotetext{
${ }^{1}$ Aside from more complex artificial shapes that have also been used but that are not necessarily inspired by hand-drawn stippling, see Sec-
} tion 2 . 


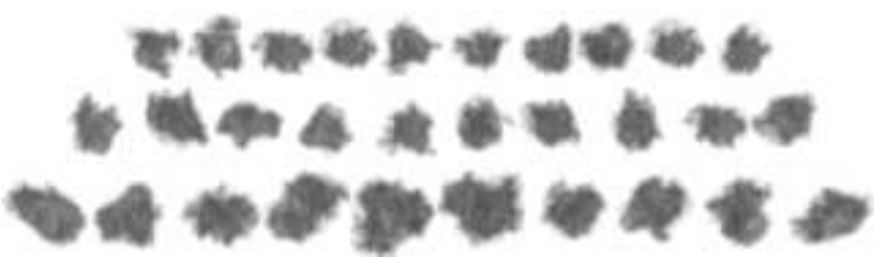

Figure 5: Enlarged hand-drawn stipple dots (scanned at $1200 \mathrm{ppi}$ ).

Figure 5. These stipple dots are not all equal but have varying shapes and sizes. One can also notice that the stipples are not completely black but do exhibit a grayscale texture. This texture is likely due to the specific interaction of the pen's ink with the paper and typically disappears when the stipple illustration is reproduced in a printing process. This lead to the assumption that stipple dots are always completely black marks on a white background as used in much of the previous literature. However, the grayscale properties of real stipple dots are a characteristic of the real stippling process which one may want to reproduce provided that one employs an appropriate reproduction technology. In addition, we also make use of these grayscale properties for realizing a technique that can address one of the remaining challenges in stippling: the merging of dots in the middle tonal ranges.

\section{A Grayscale Stippling Process}

Based on the previous analysis we now present our process for high-quality example-based stippling. In contrast to previous approaches, our process captures and maintains the stipple image throughout the entire process as highresolution grayscale image, which allows us to achieve both stipple merging for the middle tonal ranges and high-quality print output. Also, to allow for interactive control of the technique, we use Ostromoukhov's [30] fast error-diffusion halftoning technique to place the stipples. Below we step through the whole process by explaining the stipple placement (Section 4.1), the stipple dot selection and accumulation (Section 4.2), and the generation of both print and on-screen output (Section 4.3). In addition, we discuss adaptations for interactive processing (Section 4.4).

\subsection{Stipple Dot Placement using Halftoning}

Our stippling process starts by obtaining a grayscale version of the target image using the techniques described in Section 3.1. In principle, we use this image to run Ostromoukhov's [30] error-diffusion technique to derive locations for placing stipple dots, similar to the use of halftoning to determine the starting distribution in the work by Deussen et al. [17]. However, in contrast to their approach that adds point relaxation based on Lloyd's method, we use the locations derived from the halftoning directly which allows us to let stipple dots merge, unlike the results from relaxation (see Figure 6). The reason for choosing a halftoning technique over, for example,

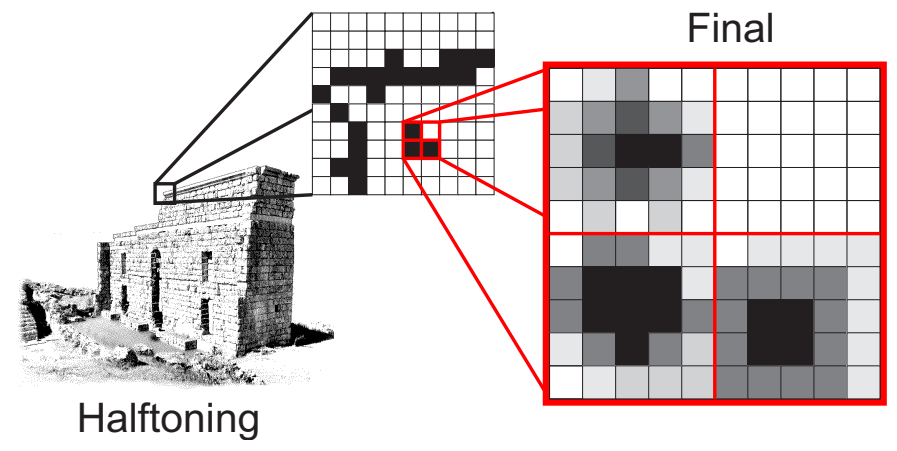

Figure 6: Basic process: one black pixel in the halftoning image is converted to one dot in the final image.

distributions based on hand-drawn stipple statistics [1] is twofold. The main reason is that halftoning has the evaluation of tone built-in so that it does not require a tone map being extracted from the hand-drawn example. The second reason lies in that halftoning provides a continuum of pixel density representing tonal changes as opposed to the approach of using both black pixels on a white background for brighter regions and white pixels on a black background for darker areas as used by Kim et al. [1].

Specifically, we are employing Ostromoukhov's [30] error-diffusion, partially because it is easy to implement and produces results at interactive frame-rates. More importantly, however, the resulting point distributions have blue noise properties, a quality also desired by related approaches [22]. This means that the result is nearly free of dot pattern artifacts that are present in results produced by many other error-diffusion techniques, a quality that is important for stippling [28].

Using a halftoning approach, however, means that we produce a point distribution based on pixels that are arranged on a regular grid, in contrast to stipples that can be placed at arbitrary positions. In addition, we cannot use the grayscale input image in the same resolution as the intended output resolution for the stipple image. Let us use an example to better explain this problem and describe its solution. Suppose we have a hand-stippled A4 image (in landscape) that we scan for analysis and extraction of stipple dot examples at 1200 ppi. $^{2}$ This means that the resulting image has roughly $14,000 \times 10,000$ pixels, with stipple dot sizes ranging from approximately $10 \times 10$ to $20 \times 20$ pixels. $^{3}$ If we now were to produce an equivalent A4 output image at $1200 \mathrm{ppi}$, would hence use an $14,000 \times 10,000$ pixel grayscale image, and compute its halftoned version at this size, each pixel in this image would represent one dot. This means we would need to place scanned stipple dots (whose average size is $16 \times 16$

\footnotetext{
${ }^{2}$ Please notice that ppi stands for pixels per inch and is used intentionally while dots per inch (dpi) is used when we discuss printing.

${ }^{3}$ These values are derived from the example in Figure 2 (done with a pen with a $0.2 \mathrm{~mm}$ tip) and can be assumed to be valid for many stipple images as similar pen sizes are used by professional illustrators [28].
} 

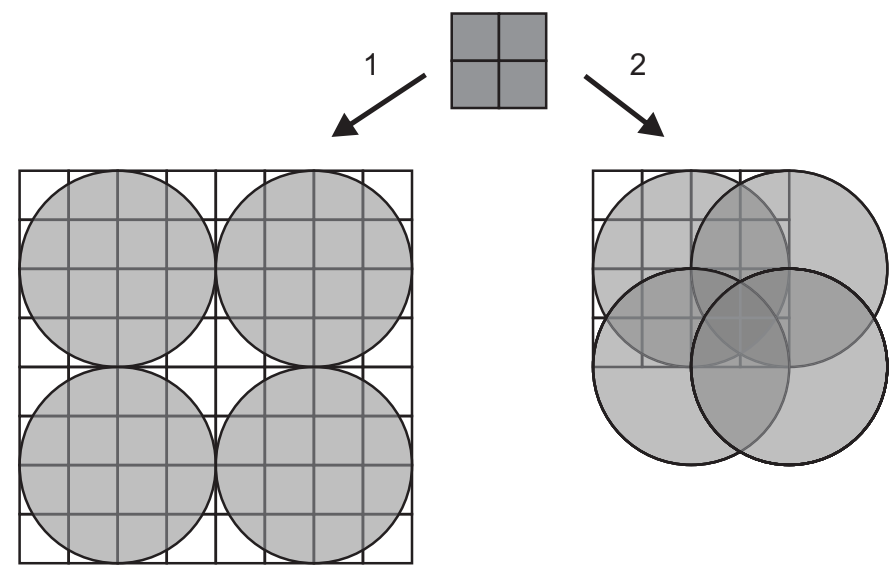

Figure 7: Effect of the packing factor.

pixels, this is equivalent to a spatial size of $0.338 \mathrm{~mm}$, slightly larger than the nominal size of $0.2 \mathrm{~mm}$ of the tip of the used pen) at the pixel locations of the halftoned image. Consequently, this would produce a result that is $16 \times$ larger than the intended output image and reproducing it at A4 size would result in the characteristics of the stipple dot shapes being lost because each stipple would again be shrunken down to the size of approximately one pixel.

Therefore, for a given output resolution $\operatorname{res}_{o}$ we compute the dot distribution using error-diffusion halftoning at a smaller halftoning resolution resht, which implies that the halftoning image must be obtained by scaling the input image. The value of the scaling is the halftoning factor $f_{h t}$. Intuitively for our 1200 ppi example, one could suggest a halftoning factor $f_{h t}$ whose value is $1 / 16$ of $r e s_{o}$ to compute $r e s_{h t}$, using the average stipple's diameter of 16 pixels:

$$
r e s_{h t}=f_{h t} \cdot r e s_{o} ; \quad f_{h t}=1 / 16 ; \quad r e s_{o}=1200 p p i
$$

In a completely black region and for ideally circular stipples, however, this would result in a pattern of white spots because the black dots on the grid only touch. To avoid this issue one has to use a factor $f_{h t}$ of $\sqrt{2} / 16$ to allow for a denser packing of stipple points such that they overlap (see Figure 7). For realistic stipple points with noncircular shapes one may even have to use a $f_{h t}$ of $2 / 16$ or more. On the other hand, even in the darkest regions in our example the stipple density is not such that they completely cover the canvas. Thus, we leave this choice up to the user to decide how dense to pack the stipples, and introduce a packing factor $f_{p}$ to be multiplied with $f_{h t}$ to control the density of the stipples:

$$
f_{h t}=f_{p} / 16 \text {. }
$$

For $f_{p}=1$ and, thus, $f_{h t}=1 / 16$ we would perform the halftoning in our example on an image with size $875 \times 625$ pixels to eventually yield a 1200 ppi landscape A4 output. For other output resolutions, however, the situation is different. Because the stipples have to use proportionally smaller pixel sizes for smaller resolutions

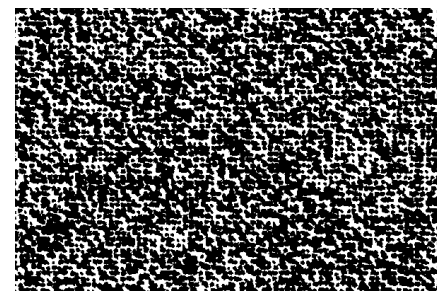

(a) Grid placement of stipples.

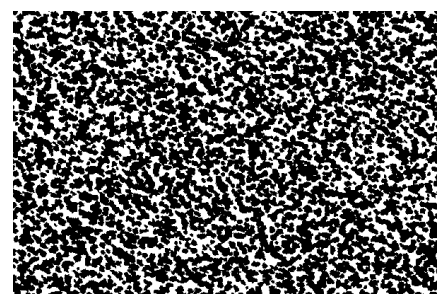

(b) After random perturbation.
Figure 8: Magnified comparison of stipple placement before and after random perturbation of the stipple locations.

to be reproduced at the same spatial sizes, the factor between output image and halftoning image has to change proportionally as well. For example, for a 600 ppi output resolution the average stipple's diameter would only be 8 pixels, and consequently $f_{h t}$ would only be $1 / 8$. Thus, we can derive the halftoning resolution res $_{h t}$ for a given output resolution res $_{o}$ in ppi based on the observations we made from scanning a sample at 1200 ppi as follows:

$$
\begin{aligned}
r e s_{h t} & =f_{h t} \cdot r e s_{o} ; \quad f_{h t}=\frac{f_{p} \cdot 1200 p p i}{16 \cdot r e s_{o}} \\
r e s_{h t} & =75 \mathrm{ppi} \cdot f_{p} .
\end{aligned}
$$

This means that the halftoning resolution is, in fact, independent of the output resolution. Consequently, the pixel size of the halftoning image only depends on the spatial size of the intended output and the chosen packing factor (and ultimately the chosen scanned example stippling whose stipple dot size depends on the used pen).

This leaves the other mentioned problem that arises from computing the stipple distribution through halftoning: the stipple dots would be arranged on a regular grid, their centers always being at the centers of the pixels from the halftoning image. To avoid this issue, we perturb the locations of the stipple dots by a random proportion of between 0 and $\pm 100 \%$ of their average diameter, in both $x$ - and $y$-direction. Together with the random selection of stipple sizes from the database of scanned stipples and the blue noise quality of the dot distribution due to the chosen halftoning technique this successfully eliminates most observable patterns in dot placement (see Figure 8).

\subsection{Stipple Dot Selection and Accumulation}

We begin the collection of computed stipple points by creating a grayscale output buffer of the desired resolution, with all pixels having been assigned the full intensity (i. e., white). Then we derive the stipple placement by re-scaling the modified grayscale image to the halftoning resolution and running the error-diffusion as described in the previous section. Based on the resulting halftoned image and the mentioned random perturbations we can now derive stipple location with respect to the full resolution of the output buffer.

For each computed location we randomly select a dot from the previously collected database of stipple dot scans. 


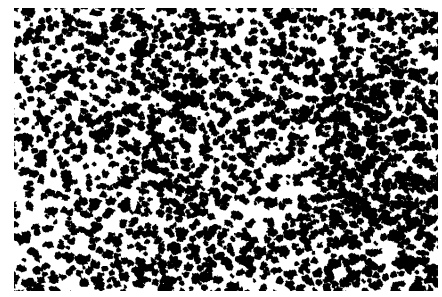

(a) Lighter region.

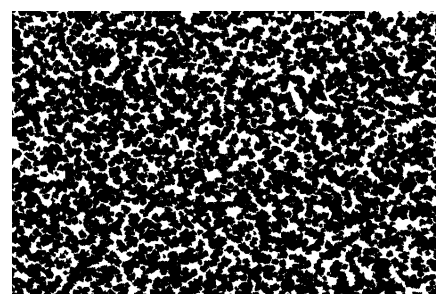

(b) Darker region.
Figure 9: Merging of synthesized stipples at two tonal ranges.

This database is organized by approximate stipple dot sizes, so that for each location we first determine the size class using a random function that follows a uniform distribution (all the sizes have the same probability) or a normal distribution centered on the average size, and then randomly select a specific dot from this class. The selection of which random function to use is of aesthetic nature and left up to the user. The default random function uses the uniform distribution, producing a less regular result. The selected dot is then added to the output buffer, combining intensities of the new stipple dot $i_{s}$ and the pixels previously placed into the buffer $i_{b g}$ using $\left(i_{s} \cdot i_{b g}\right) / 255$ (for 8 bits). This not only ensures that stipples placed on a white background are represented faithfully but also that the result gets darker if two dark pixels are combined (accumulation of ink; for example, given $i_{s}=45$ and $i_{b g}=64$, the result is 11 which is darker than the original values).

Both the range of stipple sizes and the partially random stipple placement ensure that stipples can overlap. This overlapping is essential for our approach, it ensures the gradual merging of stipples into larger conglomerates as darker tones are reproduced (see example in Figure 9). Therefore, we can for the first time simulate this aspect of the aesthetics of hand-drawn stippling.

\subsection{Generation of Print and Screen Output}

One challenge that remains is to generate the appropriate output for the intended device. Here we typically have two options: on-screen display and traditional print reproduction. These two options for output differ primarily in their spatial resolution and their color resolution. While normal bilevel printing offers a high spatial resolution (e. g., $1200 \mathrm{dpi}$ ), it has an extremely low color resolution ( 1 bit or 2 bit) while typical displays have a lower spatial resolution (approximately $100 \mathrm{ppi}$ ) but a higher color resolution (e.g., 8 bit or 256 bit per primary). These differences also affect the goals for generating stipple illustrations. For example, it does not make much sense to print a grayscale stipple image because the properties of the individual stipple points (shape, grayscale texture) cannot be reproduced by most printing technology, they would disappear behind the pattern generated by the printer's halftoning [31]. In contrast, for on-screen display it does not make sense to generate a very high-resolution image because this cannot be seen on the screen.

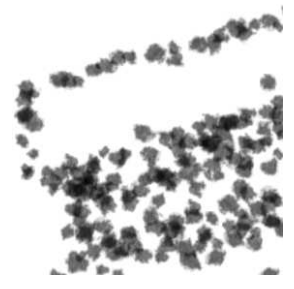

(a) Grayscale.

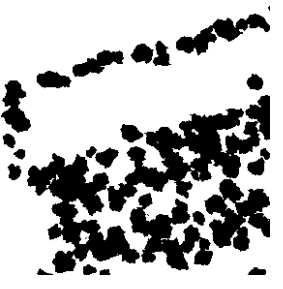

(b) Black and white.

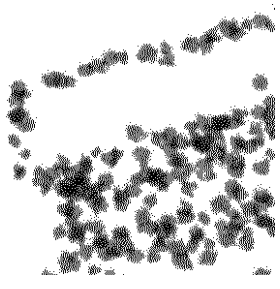

(c) Dithered.
Figure 10: Details of the 1200ppi outputs with the grayscale, black and white, and halftoning process.

Therefore, we adjust our stippling process according to the desired output resolutions, both color and spatial. For output designed for print reproduction we run the process at $1200 \mathrm{ppi}$, using a stipple library from a $1200 \mathrm{ppi}$ scan, and compute the scaling factor for the halftoning process to place the stipples accordingly. The resulting 1200 ppi grayscale output image is then thresholded using a user-controlled cut-off value, and stored as a 1 bit pixel image, ready for high-quality print at up to $1200 \mathrm{dpi}$ (e.g., Figure 16). These images, of course, do no longer contain stipples with a grayscale texture but instead are more closely related to printed illustrations in books.

As a print reproduction alternative we also provide the option to apply a dedicated halftoning step (using Ostromoukhov's [30] error-diffusion algorithm) to a grayscale stippling result. This allows us to maintain the grayscale properties of the stipple dots even in a black-and-white output medium such as print (Figure 10). For instance, for 1200 dpi output we can reproduce approximately 32 grayscale tones, while bypassing the problem that many printers apply a Postscript dithering which is limited compared to Ostromoukhov's stochastic dithering. On sufficiently high-resolution (1200 dpi and higher) printers, the dedicated dithering allows us to maintain the grayscale properties of the stipple dots while the dither pattern is close to being unnoticeable.

For on-screen display, in contrast, we run the process at a lower resolution, e. g., 300 ppi (while 300 ppi is larger than the typical screen resolution, it also allows viewers to zoom into the stipple image to some degree before seeing pixel artifacts). For this purpose the stipples in the database are scaled down accordingly, and the appropriate scaling value for the halftoning process is computed based on average stipple size at this lower resolution. The resulting image (e.g., Figure 18) is smaller spatially but we preserve the texture information of the stipples. These can then be appreciated on the screen and potentially be colored using special palettes (e.g., sepia). In addition, grayscale stipple images can also be used for special continuous tone printing processes such as dye-sublimation.

\subsection{User Interaction}

Several parameters of the process can be adjusted interactively according to aesthetic considerations of the user, in addition to applying the high-level processes (parallel 


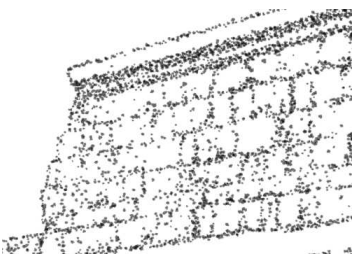

(a) Original.

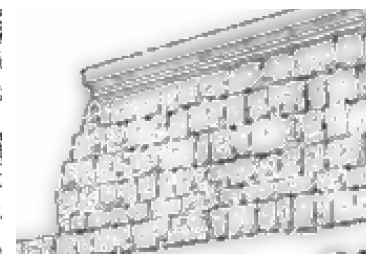

(b) DoG filter.

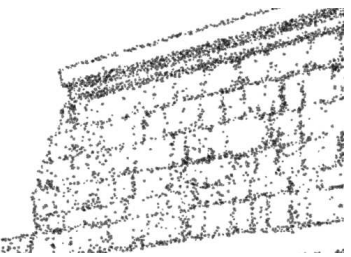

(c) Improved with DoG.

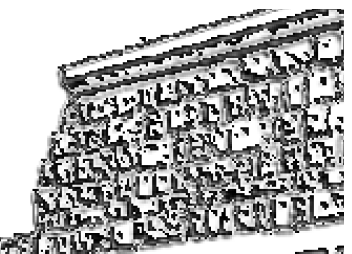

(d) Sobel filter

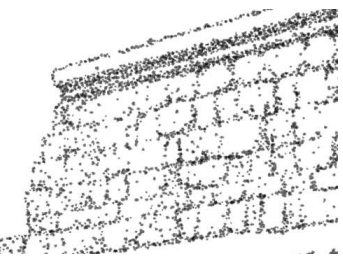

(e) Improved with Sobel.

Figure 11: Details of the improvement in the placement of borders dots (the images of the filters are scaled).

or as pre-processing). The most important settings are the intended (spatial) size and output resolution because these affect the resolutions at which the different parts of the process are performed. For example, a user would select A5 as the output size and $300 \mathrm{ppi}$ as the intended resolution. Based on this the (pixel) resolution of both output buffer and halftoning buffer are derived as outlined in Section 4.1. To control the stipple density, we let the users interactively adjust the packing factor (the default value is 2). In addition we let users control the amount of placement randomness as a percentage of the average size of the stipple dots (the default value is $25 \%$ ). This means that we specify the packing factor and placement randomness based on the average stipple size at the chosen resolution, which results in visually equivalent results regardless of which specific resolution is chosen.

\subsection{Stipple Dot Placement Improvements}

Of course, our general approach is not without flaws. One problem arises from the use of halftoning on a resolution lower than the output one to derive stipple distributions because this initially leads to the stipples being placed on a grid. While we address this grid arrangement by introducing randomness to the final stipple placement, this also leads to noise being added to otherwise clear straight lines in the input image. This effect can be observed by comparing the upper edge of the ruin in Figure 16 with the same location in the hand-made example in Figure 2 where the line of stipple dots is nicely straight.

Given the importance of lines and borders in the illustration process, we address this issue by analyzing the local character of the source image. We detect the edges in the source image using an edge detector. These border filters produce grayscale images, with darker values showing that borders are nearer. This value is used to control the random displacement of the stipple dots: the degree of the detected edge ( $d_{e}$, value between 0 and 1$)$ controls the maximum random displacement, i.e., it is used as a factor for the stipple points average diameter as used in the displacement computation. Figure 11 illustrates this displacement process, showing that reducing the displacement for stipples dots on edges reduces the noise introduced for these effects. While the improvement is subtle in these detail images, it is more visible in complete images (see the examples in Figures 20-21). This seems to be a global perception since the dots are better placed overall in the image, producing a more contrasted and defined result.

Specifically, we experimented with the Sobel operator [32] because it is fast to compute and with the Difference of Gaussians (DoG) [33] because it relates to the processing in the human perceptual system. While there is no ideal method to compute the borders to control the random displacement, the ones we implemented allow users to experiment with the effects by controlling their parameters.

The control of randomness of stipple dot placement is combined with other measures to reduce the noise on lines and borders. These measures include more control over the sizes of the stipple dots using a random selection that follows a normal distribution centered around the mean dot size to ensure that most dots on a line or border have the same mean size which maintains the aesthetic of more regularity of stipple placement on these lines.

In addition, the dot placement needs to be controlled based on the visual center of the scanned dot so that linedup dots also visually form a line. The concept of a visual center is based on the observation that, while the scanned dots are embedded in a regular and square grid, they do not have to have a regular shape or ink distribution and the geometric center of the grid is not necessarily the visual center. For example, the dot may have one main dark zone that is close to a corner, or there may be two dark zones, etc. For computing the visual center we use a straightforward approach-we 'posterize' each dot image: First, the number of gray levels is reduced to a low number of levels, usually between 4 and 7 (see Figure 12). Then, the mean position of the pixels in the darkest level is computed and recorded as the stipple dot's visual center.

All techniques used together allow us to improve the treatment of borders and lines in the stipple images but still fail to solve all issues. For example, our resolutiondependent stippling requires us to scale the input image to the size of the halftoning image, which is then subjected to the halftoning process to inform the stipple placement. The linear interpolation employed in this scaling process leads to a distribution of gray values that, after halftoning, may lead to the black pixels used for stipple dot placement not being aligned even though the original was a perfectly straight line. This produces patterns as shown in Figure 13. We leave the treatment of this problem for future work. 


\section{- 1040}

Figure 12: High resolution dots (left) and their 'posterized' versions (right) for determining the dots' visual centers.

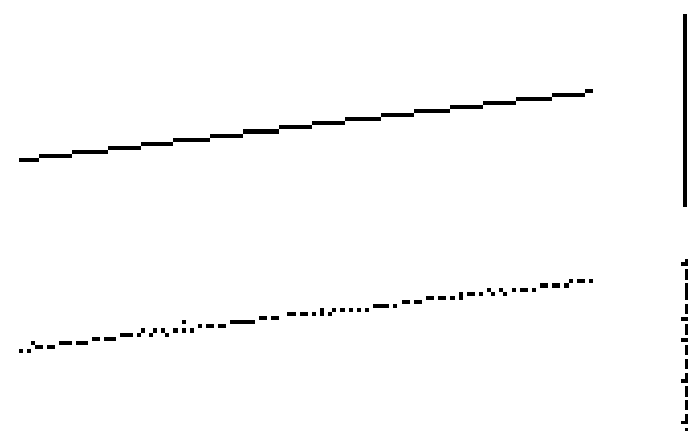

Figure 13: Scaling problem with non horizontal straight lines.

\section{Performance and GPU Implementation}

An important aspect to discuss about the stippling process is its computation and rendering performance. While we can easily allow interactive work with the CPU implementation at resolutions of up to $300 \mathrm{ppi}$ for A4 output, the process is less responsive for larger images. For example, stippling the image shown in Figure 4(c) takes approximately $0.51,0.25$, and 0.13 seconds for A4, A5, and A6 output, respectively, while a completely black input image requires $1.41,0.70$, and 0.36 seconds, respectively (Intel Core2 Duo E6600 at 3GHz with 2GB RAM, running Linux). However, our approach can easily allow users to adjust the parameters interactively at a lower resolution and then produce the final result at the intended high resolution such as for print output. For example, stippling Figure 4(c) on A4 at 1200 dpi in black-and-white takes approximately 10.1 seconds while a completely black image requires approximately 15.2 seconds. We ensure that both lowand high-resolution results are equivalent by inherently computing the same halftoning resolution for both resolutions using the resolution-dependent scaling factor and appropriately seeding the random computations.

We can improve the performance of the computation by implementing the stipple dot placement on the GPU. This is particulary useful for systems where the CPU is slower than the GPU such in some notebooks or netbooks or when the technique is used on high-resolution or large displays. For this purpose we store the library of scanned stipple points in a texture, load the halftoned image into another texture, generate a texture with random numbers due to the CPU producing better pseudo-random numbers than the GPU, and prepare a frame buffer object (FBO) to record the final output as shown in Figure 14.

We then create a fragment shader that takes over the task of placing the stipples which is the time-consuming part in the CPU implementation but which can be highly parallelized on the GPU. To create this shader we have to

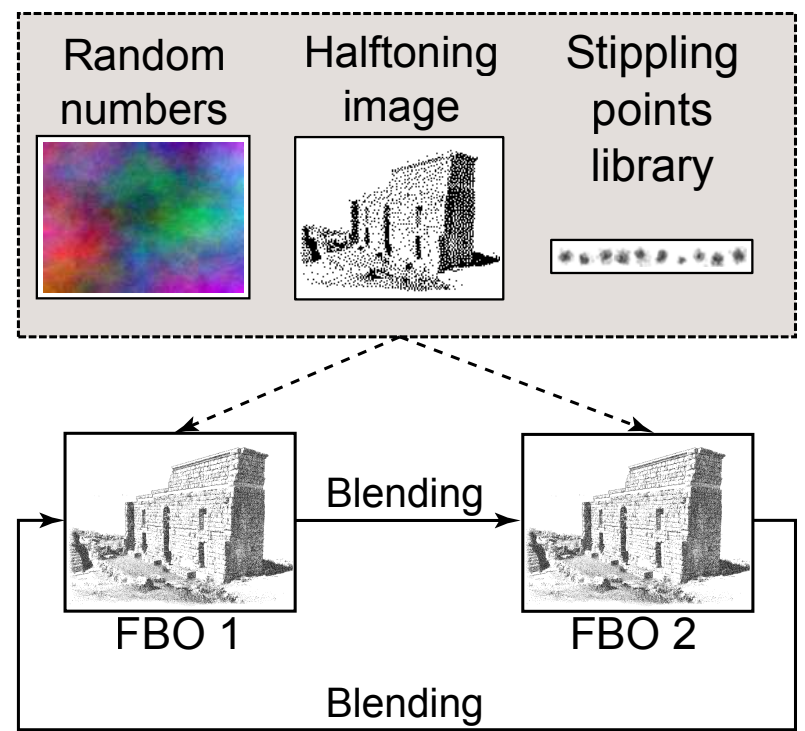

Figure 14: Three textures are passed to a fragment shader program: the halftoning, the stippling points, and a texture containing random values. The image is rendered into a frame buffer object (FBO). The partial result is blended with the previous one. Two FBOs are needed for this process because a FBO cannot feed itself.

consider that a shader only sees the fragment it is processing without being able to look into its neighborhood and that the shader is computed in parallel for many fragments at a time. The latter, in particular, means that the shader could potentially compute contributions for the same output fragment but that arise from different input fragments due to the overlapping neighboring stipple dots (from packing and random offset). However, since the fragment shaders cannot operate recursively, we need to ensure that, while placing one stipple dot, no other neighboring stipple dots are being placed. We avoid the processing of overlapping stipple dots by only computing dots on a regular grid in one pass, spaced apart in both the horizontal and the vertical direction (see Figure 15). The spacing between concurrently processed dots depends on the maximum dot size, the maximum random displacement, and the packing factor, and the scaling factor. Specifically, the minimal separation for the dots is computed as the size of the larger dot divided (integer division) by the average size of the dot and then multiplied by the average size of the dot plus twice the average size of the dot. Then, the process/pass is repeated by repeatedly moving the grid to the next untreated set of stipple dots until all potential dots have been placed. For example, the largest dot in our dot library for $1200 \mathrm{dpi}$ b/w images has a size of $56^{2}$ pixels, while the average size is $24^{2}$ pixels. Thus, the separation is computed as sep $=\left(\right.$ ceil $\left(\right.$ size largest_dot $_{\text {d }} /$ size $\left.\left._{\text {average_dot }}\right)+2\right)$. size $_{\text {average_dot }}=($ ceil $(56 / 24)+2) \cdot 24=5 \cdot 24$ to be 120 pixels. The number of passes per coordinate axis depends on how dense the stipple points lie to each other, which is controlled by the packing factor $f_{p}$. The total number of passes, therefore, can be computed as passes $=($ floor $($ sep . $f_{p} /$ size $\left.\left._{\text {average_dot } t}\right)\right)^{2}$. Hence, in our example we have to compute 100 passes of the shader code using a packing 


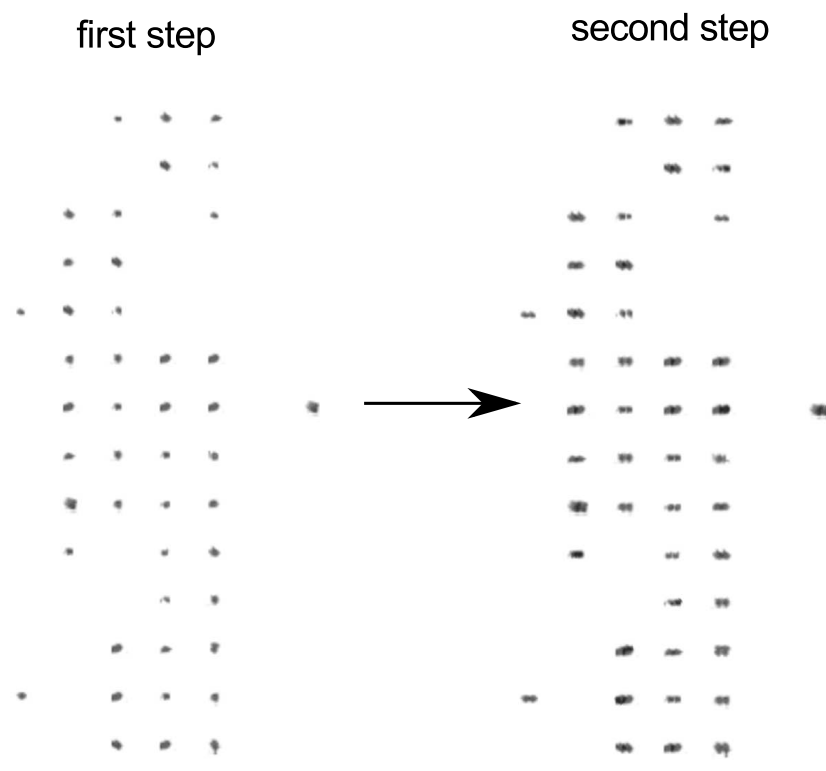

Figure 15: The two first steps of the algorithm: In the $1^{\text {st }}$ step (left), the algorithm renders the dots with the necessary distance to prevent overlapping. In the $2^{\text {nd }}$ step (right), the next dots are rendered and blended to the previous step. The algorithm is repeated until the final illustration is complete.

factor of 2. An example of the two first steps of this algorithm is shown in Figure 15. ${ }^{4}$

To ease the actual stipple dot placing, we actually scale the halftoning input image during pre-processing without interpolation (NEAREST filter) to avoid repeated position calculations. During processing, the shader determines if it is treating an active grid location and checks for a black pixel in the scaled halftoning texture. If this is the case, the shader determines the center location of the stipple point currently being treated, the pixel offset of the fragment being considered to the center of the dot, with this derives the stipple dot texel, and determines the overall offset for the current stipple dot. Based on this computation, the gray level of the output fragment is being determined and blended with the previous fragment at this location. For this purpose we use two FBOs in an alternating setup to avoid feedback loops. This means that the newly computed fragment is blended with the previous fragment at this location (from $\mathrm{FBO}_{2}$ ) and is placed into $\mathrm{FBO}_{1}$. If no new fragment is computed (not on active grid or halftoning pixel not black) the fragment from $\mathrm{FBO}_{2}$ is simply copied into $\mathrm{FBO}_{1}$. After each pass, the pointers to both FBOs are swapped. The result of the GPU algorithm is exactly the same as the basic CPU algorithm only if the random values are stored in the same order and if the blending operation were commutative (which is the case of our CPU implementation). Thus, the difference between both algorithms is the order of rendering because the GPU algorithm chooses the dots that do not overlap in

\footnotetext{
${ }^{4}$ The detailed algorithm can also be reviewed in the shader code of the demo.
}

the same pass, and then blending them with a previous set of dots. The main limitation with respect to the CPU algorithm lies in the memory limits of the GPU because the result is captured in the two FBOs, thus the GPU technique is limited with respect to the image sizes it can process.

The performance of the algorithm depends on the parallelization of the dots rendering. If the packing factor is small (i.e., 1-3), the algorithm will work very fast (i.e., approx. $100 \mathrm{fps}$ for an A5 image at $1200 \mathrm{ppi}$ on a GeForce 9400 GT) because grid spacing is small and thus the number of passes will be small (i.e., approx. 121). The reason that 121 passes are still rendered very fast is that the shader is relatively simple and that each pass only needs to render a single, screen-filling quad. In addition, the fragment shader used does not contain any loops so the operations are executed very fast for every fragment. However, if the packing factor is high (e.g., approx. 6), the number of passes will increase (approx. 529 in this case), thus losing performance (approx. $5 \mathrm{fps}$ for the same image). Nevertheless, the algorithm on the GPU is faster than on the CPU because the number of black stipple dots in the halftoning image has almost no affect on the performance of the GPU algorithm.

\section{Results and Discussion}

Figure 16 shows a synthesized stipple image based on the photo (Figure 3(a)) in its touched-up form (Figure 4(c)) that was also used to create the hand-drawn example in Figure 2. Figure 16 was produced for print-reproduction at A5 and $1200 \mathrm{dpi}$. As can be seen from Figure 17, our process can nicely reproduce the merging of stipples in a way that is comparable between the hand-drawn example and the computer-generated result. In addition, this process preserves the characteristic stipple outlines found in hand-drawn illustrations.

Figure 18 shows an example produced for on-screen viewing. In contrast to the black-and-white image in Figure 16, this time the grayscale texture of the stipple dots is preserved. In fact, in this example we replaced the grayscale color palette with a sepia palette to give the illustration a warmer tone, a technique that is often associated with aging materials. However, in typical print processes, images like this will be reproduced with halftoning to depict the gray values. These halftoning patterns typically 'fight' with the stipple shapes and placement patterns. To avoid these, one has to produce $\mathrm{b} / \mathrm{w}$ output as discussed before by employing thresholding (or through stochastic dithering if the printer has a sufficiently high resolution) or use dye-sublimation printing which can reproduce gray values.

Figures 20-22 show examples with improved stipple placement using edge detectors. In particular Figure 22 exhibits an improved stipple placement which is visible, for example, at the edges of windows and other dark zones. For comparison, Figure 23 shows the higher-resolution black-and-white version of Figure 22. 


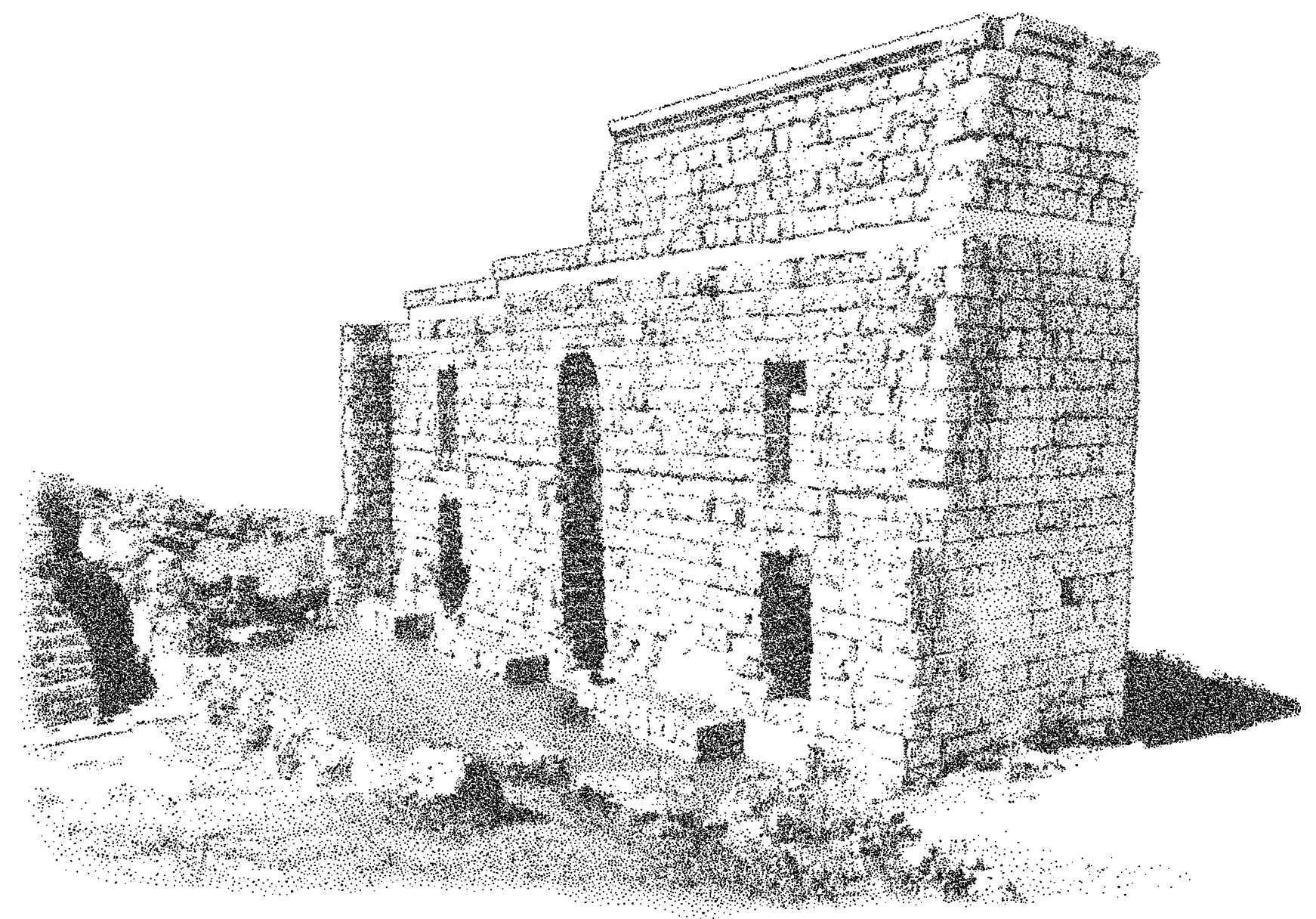

Figure 16: Example generated for A4 print reproduction at 1200 dpi resolution, using Figure 4(c) as input.

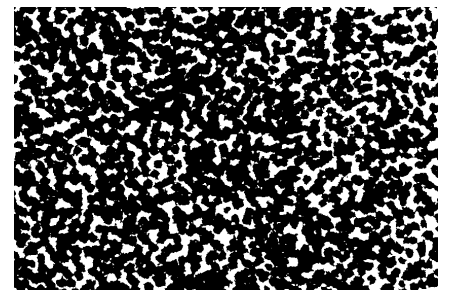

(a) Detail from Figure 2.

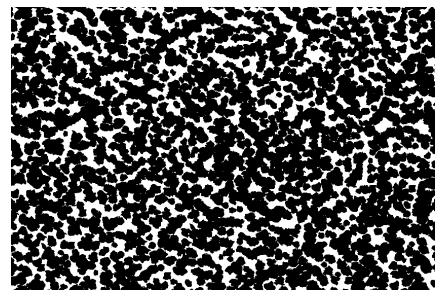

(b) Detail from Figure 16.

Figure 17: Comparison of stipple merging between a hand-drawn and a synthesized sample, taken from the same region of the images.

One final aspect that we would like to discuss is the use of a halftoning image as the basis for the stipple placement. While we have presented the reasons for our decision in Section 4.1, one may also argue that it could be better to use other types of halftoning (e.g., [34]) or try to adapt Kim et al.'s [1] technique to fit our needs. To investigate this issue further, we took samples from both Figures 2 and 16 and analyzed these using the statistical approach described by Maciejewski et al. [27]. The result of this analysis is shown in Figure 19 and reveals that the examined hand-drawn and our computer-generated stippling examples exhibit almost identical statistical behavior when compared with each other. Following the lead by Maciejewski et al. [27],

we compared the samples with respect to the correlation, energy, and contrast measures. We can see in Figure 19 that the the curves for related regions closely match each other, regardless if the sample was taken from the handdrawn image or the computer-generated one. In addition, the curves representing the two distinct regionsdensely stippled and sparsely stippled-also match each other quite closely. Finally, the graphs show that our computer-generated examples do not exhibit the correlation artifacts described by Maciejewski et al. [27] for other computer-generated stippling techniques. Thus, we can conclude that our choice to base the stipple distribution on halftoning seems to be justified.

\section{Conclusion and Future Work}

In summary, we presented a scale-dependent, examplebased stippling technique that supports both low-level stipple placement and high-level interaction with the stipple illustration. In our approach we employ halftoning for stipple placement and focus on the stipples' shape and texture to produce both gray-level output for on-screen viewing and high-resolution binary output for printing. By capturing and maintaining the stipple dots as grayscale textures throughout the process we solve the problem of 


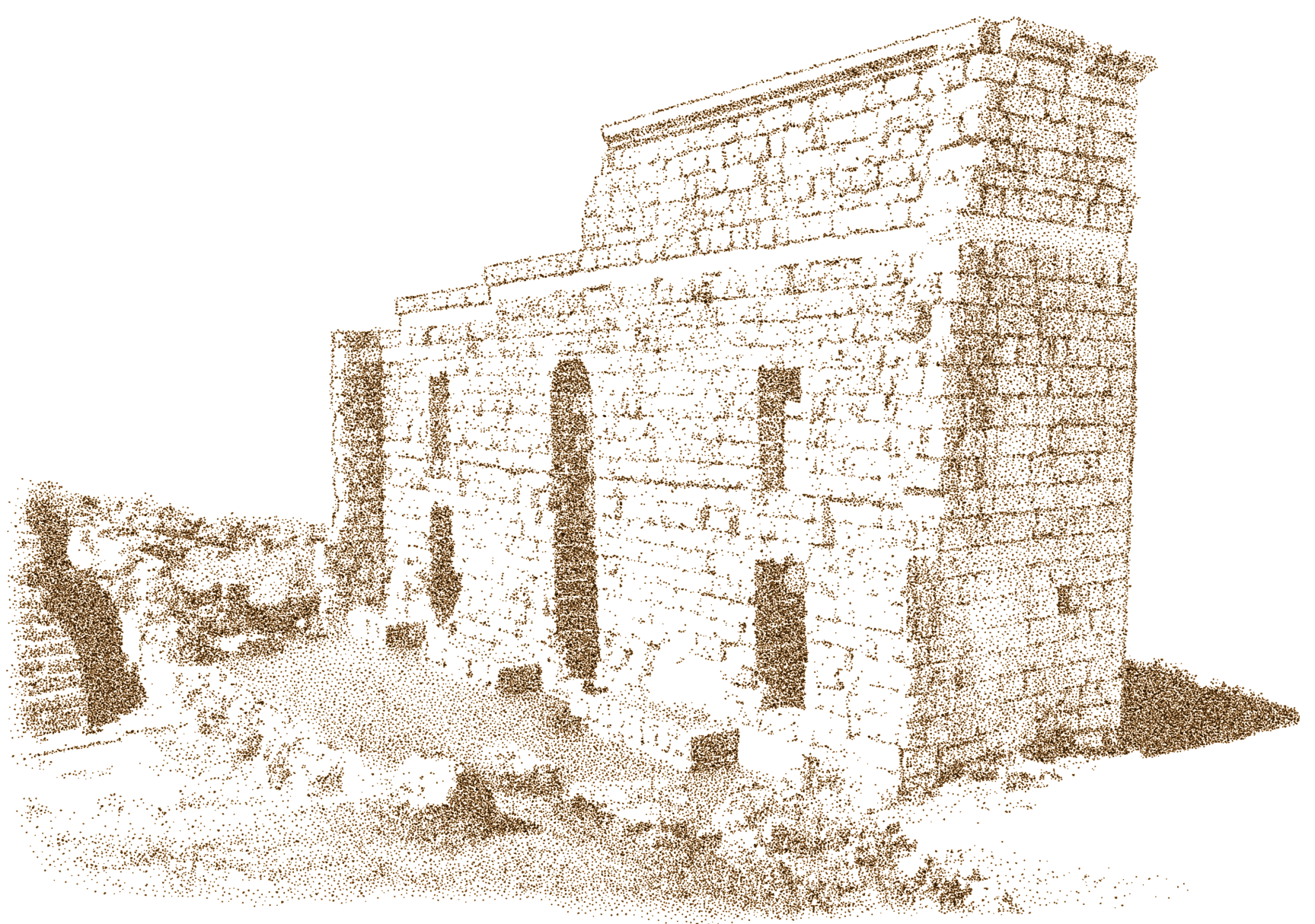

Figure 18: Sepia tonal stipple example generated for A4 on-screen viewing at up to 300 ppi resolution, with slight gamma correction.

the merging of stipple dots at intermediate resolutions as previously reported by Kim et al. [1]. The combined technique allows us to capture the entire process from artistic and presentation decisions of the illustrator to the scale-dependence of the produced output. We discuss both CPU and GPU implementations of the technique, the CPU version allowing us to add further control while the GPU version can be run at real-time frame-rates for better interactive experimentation with the parameters.

One of the interesting observations from our stippling process is that the resolution at which the stipple distribution occurs (using halftoning in our case) depends on the spatial size of the target image but needs to be independent from its resolution, just like other pen-andink rendering [35, 36, 37]. For example, there should be the same number of stipples for a 1200 ppi printer as there should be for a 100 ppi on-screen display. However, there need to be fewer stipples for an A6 image compared to an A4 image. This complements the observation by Isenberg et al. [26] that stippling with many dense stipple points is often perceived by viewers to be computer-generated.

While our approach allows us to support the interactive creation of stipple illustrations, this process still has a number of limitations. One of the limitations we mentioned previously consists of issues concerning stipple placement at edges in the input image, where edges in the image would become fuzzy due to the introduced randomness. We partially solved this issue by controlling the amount of randomness when placing the stipples, depending on the results of an edge filter. However, the scaling involved in our scale-dependent stippling process combined with halftoning as the underlying placement principle still results in some lines not being as crisp as would be desired. One of the most important remaining limitations concerns the presentation of the interaction: the use of high-level processes as described in Section 3.1 is currently a separate process that does require knowledge of the underlying artistic principles-a better integration of this procedure into the user interface would be desirable. Also, we would like to investigate additional algorithmic support for these high-level interaction. This includes, for example, an advanced color-to-gray conversion techniques $[38,39,40]$ to support illustrators in their work. In addition, an interactive or partially algorithmically supported creation of layering or image sections according to the discussed high-level criteria such as background, low or high detail, level of contrast, or inversion would be interesting to investigate as future work. For this automatic or saliencebased abstraction techniques [41] could be employed. 
(a) Samples: original-dense (same as Figure 17(a)), syntheticdense (same as Figure 17(b)), original-sparse from Figure 2, and synthetic-sparse from Figure 16, respectively.

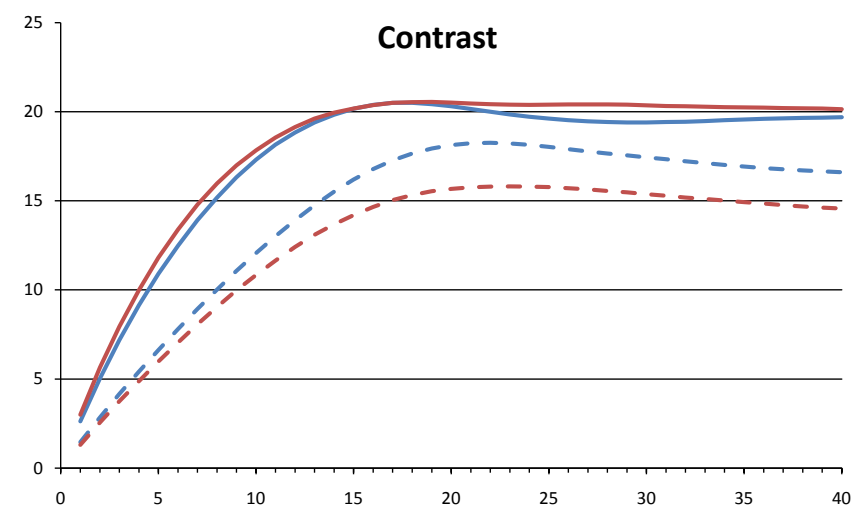

(b) original-dense $\longrightarrow$ synthetic-dense - original-sparse - - synthetic-sparse

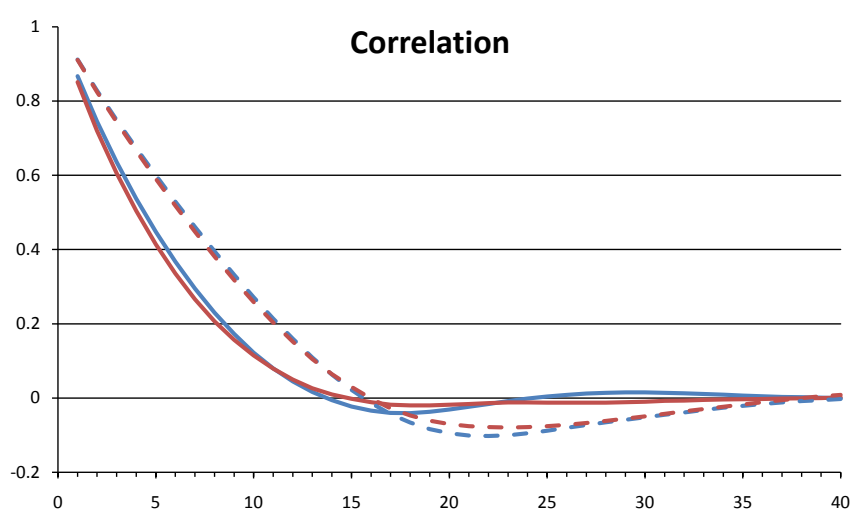

(c) original-dense $\longrightarrow$ synthetic-dense - - original-sparse - - synthetic-sparse

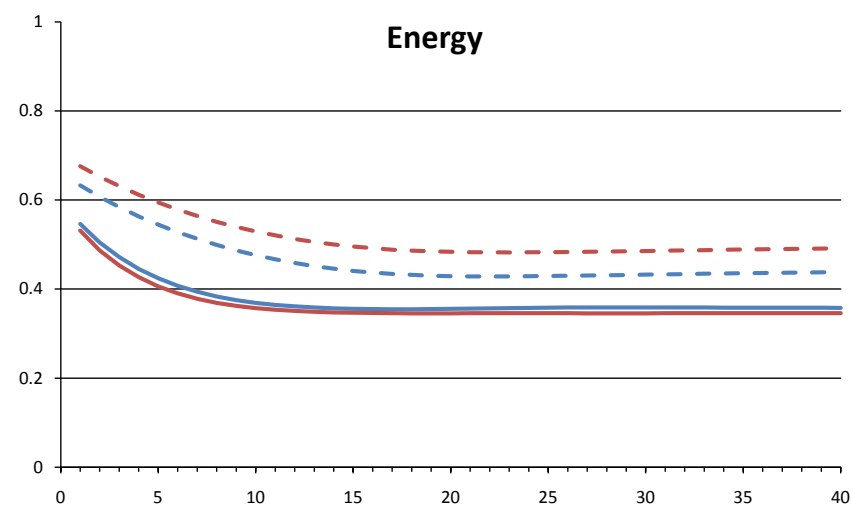

(d) original-dense $\longrightarrow$ synthetic-dense - - original-sparse - - synthetic-sparse

Figure 19: Samples and results of the statistical evaluation.

\section{Acknowledgments}

We thank, in particular, Elena Piñar for investing her time and creating the stippling examples for us. We also thank Ross Maciejewski who provided the stipple statistics script and Moritz Gerl for his help with Matlab, and the reviewers for their nice suggestions on improving the paper. Finally, we acknowledge the support of the Spanish Ministry of Education and Science to the projects TIN2007-67474-C03-02 and TIN2007-67474-C03-01, and the Ministry of Innovation, Science, and Business of the Junta de Andalucía to the project PE09-TIC-5276.

\section{References}

[1] Kim S, Maciejewski R, Isenberg T, Andrews WM, Chen W, Costa Sousa M, et al. Stippling By Example. In: Proc. NPAR. New York: ACM; 2009, p. 41-50. doi: 10.1145/1572614.1572622

[2] Martín D, Arroyo G, Luzón MV, Isenberg T. Example-Based Stippling using a Scale-Dependent Grayscale Process. In: Proc. NPAR. New York: ACM; 2010, p. 51-61. doi: 10.1145/1809939.1809946

[3] Lu A, Morris CJ, Ebert DS, Rheingans P, Hansen C. NonPhotorealistic Volume Rendering using Stippling Techniques. In: Proc. VIS. Los Alamitos: IEEE Computer Society; 2002, p. 211-8. doi: 10.1109/VISUAL.2002.1183777

[4] Lu A, Morris CJ, Taylor J, Ebert DS, Hansen C, Rheingans P, et al. Illustrative Interactive Stipple Rendering. IEEE Transactions on Visualization and Computer Graphics 2003;9(2):127-38. doi: 10.1109/TVCG.2003.1196001

[5] Foster K, Jepp P, Wyvill B, Costa Sousa M, Galbraith C, Jorge JA. Pen-and-Ink for BlobTree Implicit Models. Computer Graphics Forum 2005;24(3):267-76. doi: 10.1111/j.1467-8659.2005.00851.x

[6] Schmidt R, Isenberg T, Jepp P, Singh K, Wyvill B. Sketching, Scaffolding, and Inking: A Visual History for Interactive 3D Modeling. In: Proc. NPAR. New York: ACM; 2007, p. 23-32. doi: 10.1145/1274871.1274875

[7] Xu H, Chen B. Stylized Rendering of 3D Scanned Real World Environments. In: Proc. NPAR. New York: ACM; 2004, p. 25-34. doi: $10.1145 / 987657.987662$

[8] Zakaria N, Seidel HP. Interactive Stylized Silhouette for PointSampled Geometry. In: Proc. GRAPHITE. New York: ACM; 2004, p. 242-9. doi: 10.1145/988834.988876

[9] Lu A, Taylor J, Hartner M, Ebert DS, Hansen CD. HardwareAccelerated Interactive Illustrative Stipple Drawing of Polygonal Objects. In: Proc. VMV. Aka GmbH; 2002, p. 61-8.

[10] Costa Sousa M, Foster K, Wyvill B, Samavati F. Precise Ink Drawing of 3D Models. Computer Graphics Forum 2003;22(3):369-79. doi: 10.1111/1467-8659.00684

[11] Meruvia Pastor OE, Freudenberg B, Strothotte T. Real-Time Animated Stippling. IEEE Computer Graphics and Applications 2003;23(4):62-8. doi: 10.1109/MCG.2003.1210866

[12] Vanderhaeghe D, Barla P, Thollot J, Sillion FX. Dynamic Point Distribution for Stroke-based Rendering. In: Rendering Techniques. Aire-la-Ville, Switzerland: Eurographics Association; 2007, p. 13946. doi: 10.2312/EGWR/EGSR07/139-146

[13] Yuan X, Nguyen MX, Zhang N, Chen B. Stippling and Silhouettes Rendering in Geometry-Image Space. In: Proc. EGSR. Aire-la-Ville, Switzerland: Eurographics Association; 2005, p. 193-200. doi: 10. 2312/EGWR/EGSR05/193-200

[14] Hertzmann A. A Survey of Stroke-Based Rendering. IEEE Computer Graphics and Applications 2003;23(4):70-81. doi: 10.1109/MCG. 2003.1210867

[15] Lloyd SP. Least Squares Quantization in PCM. IEEE Transactions on Information Theory 1982;28(2):129-37.

[16] McCool M, Fiume E. Hierarchical Poisson Disk Sampling Distributions. In: Proc. Graphics Interface. San Francisco: Morgan Kaufmann Publishers Inc.; 1992, p. 94-105.

[17] Deussen O, Hiller S, van Overveld C, Strothotte T. Floating Points: A Method for Computing Stipple Drawings. Computer Graphics Forum 2000;19(3):41-50. doi: 10.1111/1467-8659.00396

[18] Secord A. Weighted Voronoi Stippling. In: Proc. NPAR. New York: ACM; 2002, p. 37-43. doi: 10.1145/508530.508537 
[19] Schlechtweg S, Germer T, Strothotte T. RenderBots-Multi Agent Systems for Direct Image Generation. Computer Graphics Forum 2005;24(2):137-48. doi: 10.1111/j.1467-8659.2005.00838.x

[20] Mould D. Stipple Placement using Distance in a Weighted Graph. In: Proc. CAe. Aire-la-Ville, Switzerland: Eurographics Assoc.; 2007, p. 45-52. doi: 10.2312/COMPAESTH/COMPAESTH07/045 $-052$

[21] Kim D, Son M, Lee Y, Kang H, Lee S. Feature-Guided Image Stippling. Computer Graphics Forum 2008;27(4):1209-16. doi: 10.1111/j.1467-8659.2008.01259.x

[22] Kopf J, Cohen-Or D, Deussen O, Lischinski D. Recursive Wang Tiles for Real-Time Blue Noise. ACM Transactions on Graphics 2006;25(3):509-18. doi: 10.1145/1141911.1141916

[23] Hiller S, Hellwig H, Deussen O. Beyond Stippling - Methods for Distributing Objects on the Plane. Computer Graphics Forum 2003;22(3):515-22. doi: 10.1111/1467-8659.00699

[24] Secord A, Heidrich W, Streit L. Fast Primitive Distribution for Illustration. In: Proc. EGWR. Aire-la-Ville, Switzerland: Eurographics Association; 2002, p. 215-26. doi: 10.1145/581924.581924

[25] Dalal K, Klein AW, Liu Y, Smith K. A Spectral Approach to NPR Packing. In: Proc. NPAR. New York: ACM; 2006, p. 71-8. doi $10.1145 / 1124728.1124741$

[26] Isenberg T, Neumann P, Carpendale S, Costa Sousa M, Jorge JA Non-Photorealistic Rendering in Context: An Observational Study. In: Proc. NPAR. New York: ACM; 2006, p. 115-26. doi: 10.1145/ 1124728.1124747

[27] Maciejewski R, Isenberg T, Andrews WM, Ebert DS, Costa Sousa M, Chen W. Measuring Stipple Aesthetics in Hand-Drawn and Computer-Generated Images. IEEE Computer Graphics and Applications 2008;28(2):62-74. doi: 10.1109/MCG.2008.35

[28] Hodges ERS, editor. The Guild Handbook of Scientific Illustration. Hoboken, NJ, USA: John Wiley \& Sons; $2^{\text {nd }}$ ed.; 2003. ISBN 0-47136011-2.

[29] Guptill AL. Rendering in Pen and Ink. New York: Watson-Guptill Publications; 1997.

[30] Ostromoukhov V. A Simple and Efficient Error-Diffusion Algorithm. In: Proc. SIGGRAPH. New York: ACM; 2001, p. 567-72. doi: 10 . $1145 / 383259.383326$

[31] Isenberg T, Carpendale MST, Costa Sousa M. Breaking the Pixel Barrier. In: Proc. CAe. Aire-la-Ville, Switzerland: Eurographics Association; 2005, p. 41-8. doi: 10.2312/COMPAESTH/ COMPAESTH05/041-048

[32] Sobel I, Feldman G. A $3 \times 3$ Isotropic Gradient Operator for Image Processing. Talk at Stanford Artificial Project; 1968.

[33] Marr D, Hildreth E. Theory of Edge Detection. Proceedings of the Royal Society B: Biological Sciences 1980;207(1167):187-217. doi: 10.1098/rspb.1980.0020

[34] Chang J, Alain B, Ostromoukhov V. Structure-Aware ErrorDiffusion. ACM Transactions on Graphics 2009;28(5):162(1-8). doi: 10.1145/1618452.1618508

[35] Salisbury MP, Anderson C, Lischinski D, Salesin DH. ScaleDependent Reproduction of Pen-and-Ink Illustration. In: Proc. SIGGRAPH. New York: ACM; 1996, p. 461-8. doi: 10.1145/ 237170.237286

[36] Jeong K, Ni A, Lee S, Markosian L. Detail Control in Line Drawings of 3D Meshes. The Visual Computer 2005;21(8-10):698-706. doi: 10.1007/s00371-005-0323-1

[37] Ni A, Jeong K, Lee S, Markosian L. Multi-Scale Line Drawings from 3D Meshes. In: Proc. I3D. New York: ACM; 2006, p. 133-7. doi: 10.1145/1111411.1111435

[38] Gooch AA, Olsen SC, Tumblin J, Gooch B. Color2Gray: SaliencePreserving Color Removal. In: Proc. SIGGRAPH. New York: ACM; 2005, p. 634-9. doi: 10.1145/1186822.1073241

[39] Rasche K, Geist R, Westall J. Detail Preserving Reproduction of Color Images for Monochromats and Dichromats. IEEE Computer Graphics and Applications 2005;25(3):22-30. doi: 10.1109/MCG. 2005.54

[40] Rasche K, Geist R, Westall J. Re-Coloring Images for Gamuts of Lower Dimension. Computer Graphics Forum 2005;24(3):423-32. doi: 10.1111/j.1467-8659.2005.00867.x

[41] Santella A, DeCarlo D. Visual Interest and NPR: An Evaluation and
Manifesto. In: Proc. NPAR. New York: ACM; 2004, p. 71-150. doi: $10.1145 / 987657.987669$ 


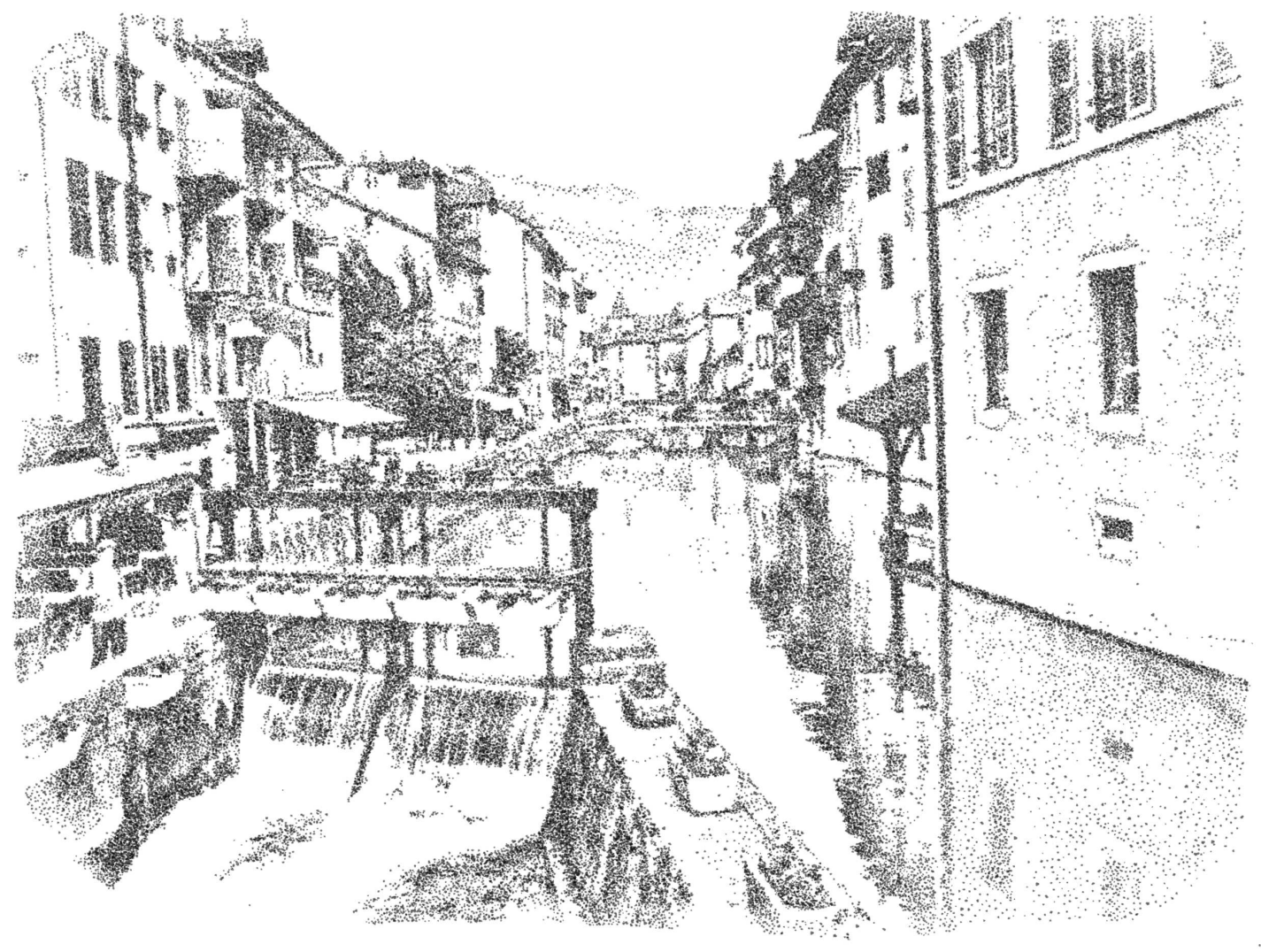

Figure 20: A5 300 ppi grayscale stippling of Annecy without enhancing the borders.

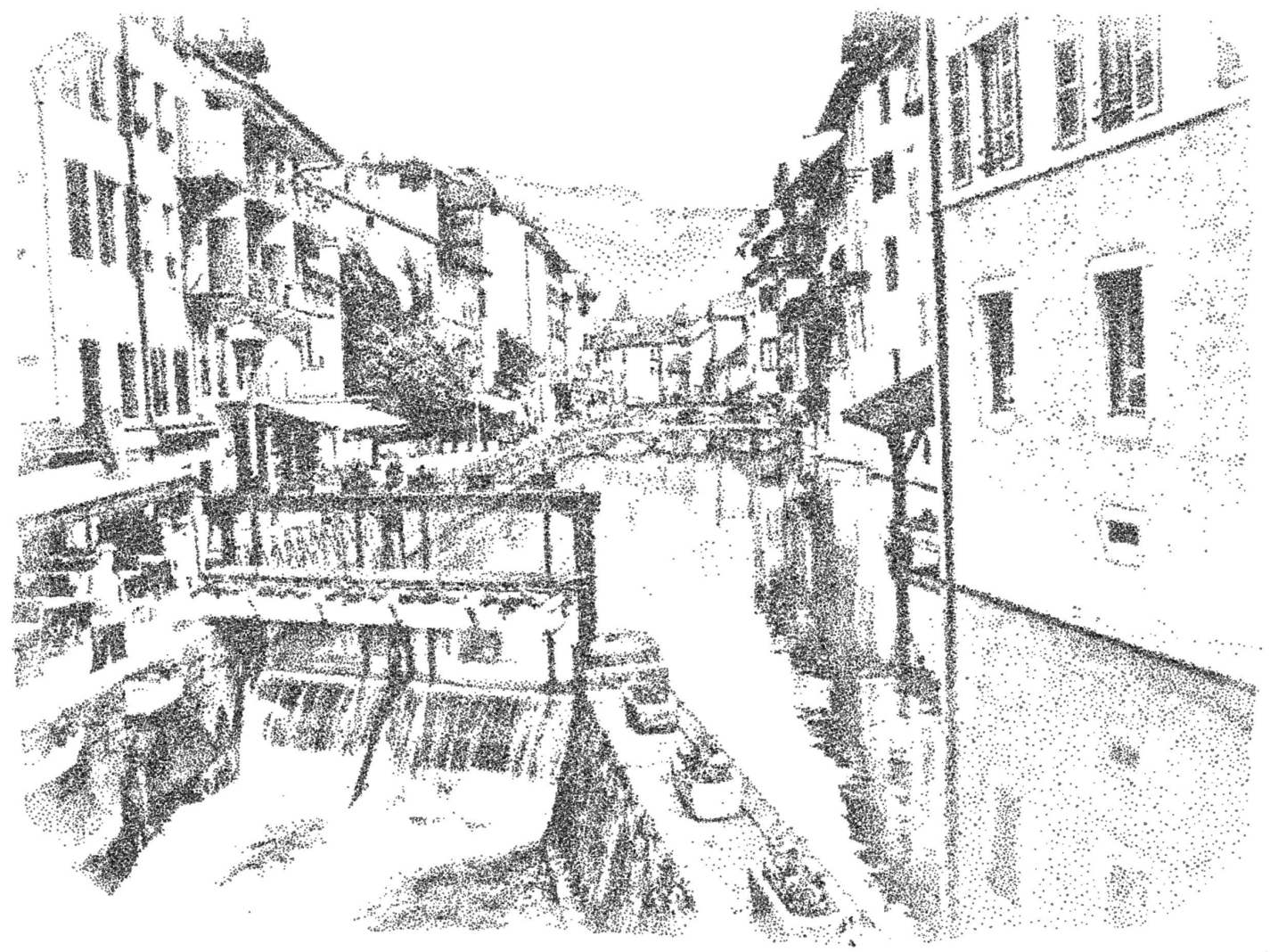

Figure 21: The same example enhanced with a Sobel filter. 


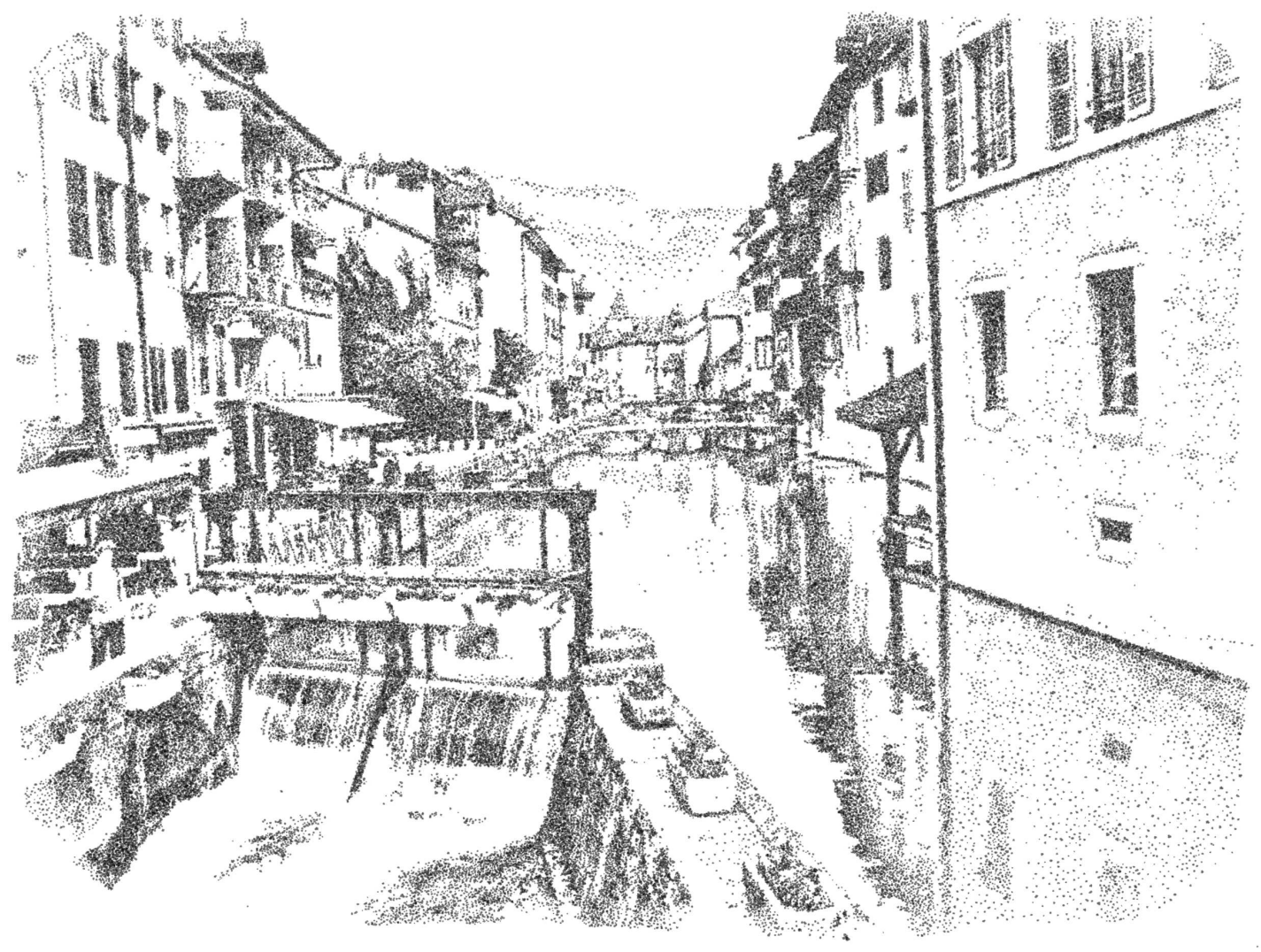

Figure 22: The same example enhanced with a DoG filter.

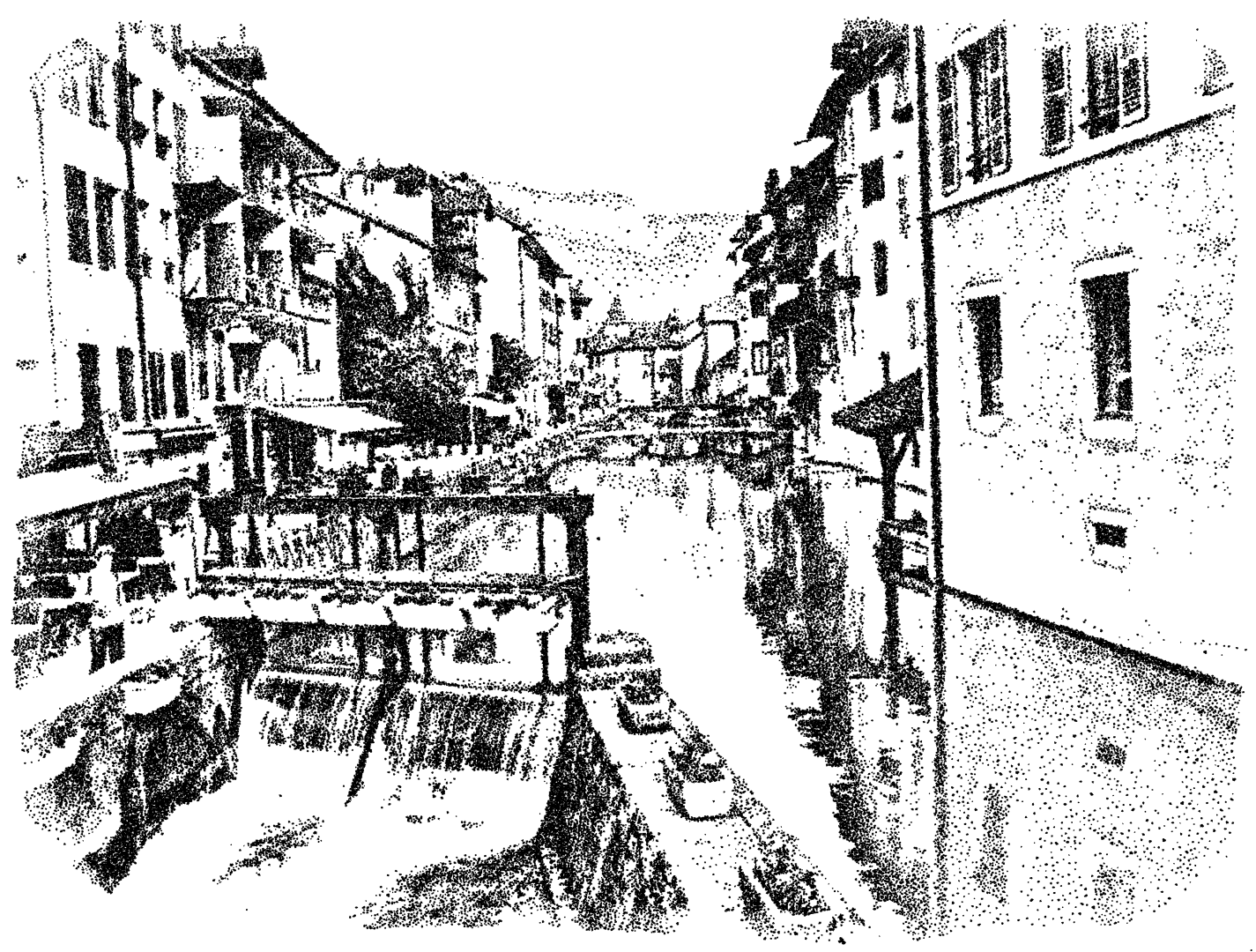

Figure 23: The 1200 ppi black-and-white version of Figure 22. 